\title{
Associative convolutions arising from conditionally free convolution
}

\author{
Anna Krystek, Łukasz Wojakowski* \\ Mathematical Institute \\ University of Wrocław \\ pl.Grunwaldzki $2 / 4$ \\ 50-384 Wrocław, Poland \\ Anna.Krystek@math.uni.wroc.pl \\ Lukasz.Wojakowski@math.uni.wroc.pl
}

December 22, 2004

\begin{abstract}
We define two families of deformations of probability measures depending on the second free cumulants and the corresponding new associative convolutions arising from the conditionally free convolution. These deformations do not commute with dilation of measures, which means that the limit theorems cannot be obtained as a direct application of the theorems for the conditionally free case. We calculate the general form of the central and Poisson limit theorems. We also find the explicit form for three important examples.
\end{abstract}

AMS Subject Classification: Primary: 46L53, 46L54, Secondary: 60E10

Keywords: Convolution, conditionally free, deformations, moment-cumulant formulae, limit theorems.

\section{Introduction}

In the paper [BLS] by Bożejko, Leinert and Speicher the notion of conditionally free convolution of pairs of compactly supported probability measures on $\mathbb{R}$ was introduced. For pairs of probability measures $\left(\mu_{1}, \nu_{1}\right),\left(\mu_{2}, \nu_{2}\right)$ their conditionally free convolution is a pair of measures

$$
(\xi, \eta)=\left(\mu_{1}, \nu_{1}\right) \square\left(\mu_{2}, \nu_{2}\right)
$$

where $\eta=\nu_{1} \boxplus \nu_{2}$ is the Voiculescu's free convolution of the measures $\nu_{1}$ and $\nu_{2}$.

Let $\mu$ be a compactly supported probability measure. By $m_{\mu}(n)$ we denote its $n$-th moment, by $r_{\mu}(n)-$ its $n$-th free cumulant and by $R_{\mu}(z)$ its Voiculescu $R$ transform where $z$ is in some neighbourhood of zero. $R$ and $r$ are related by $R_{\mu}(z)=\sum_{n=1}^{\infty} r_{\mu}(n) z^{n-1}$. For a pair of measures $(\mu, \nu)$ by $R_{(\mu, \nu)}(n)$ and $R_{(\mu, \nu)}(z)$ we denote its $n$-th conditionally free cumulant and conditionally free $R$ transform, respectively.

The conditionally free convolution of the pairs of measures $(\mu, \nu)=\left(\mu_{1}, \nu_{1}\right) 凹\left(\mu_{2}, \nu_{2}\right)$ is defined by the requirement that both the free cumulants of the measures $\nu_{i}$ and the conditionally free cumulants of the pair $\left(\mu_{i}, \nu_{i}\right)$ behave additively, that is

$$
\begin{aligned}
r_{\nu}(n) & =r_{\nu_{1}}(n)+r_{\nu_{2}}(n), \\
R_{(\mu, \nu)}(n) & =R_{\left(\mu_{1}, \nu_{1}\right)}(n)+R_{\left(\mu_{2}, \nu_{2}\right)}(n) .
\end{aligned}
$$

\footnotetext{
${ }^{1}$ Partially sponsored with KBN grant no 2P03A00723 and RTN HPRN-CT-2002-00279.

The authors wish to thank Marek Bożejko and Wojciech Młotkowski for useful suggestions.
} 
Moreover, the resulting cumulants $r_{\nu}(n)$ and $R_{(\mu, \nu)}(n)$ determine the measures $(\mu, \nu)$ uniquely. The above equations can be written in terms of the corresponding transforms $R_{\mu}(z)$ and $R_{(\mu, \nu)}(z)$, which in turn can be found by the equations

$$
\begin{aligned}
& \frac{1}{G_{\nu}(z)}=z-R_{\nu}\left(G_{\nu}(z)\right), \\
& \frac{1}{G_{\mu}(z)}=z-R_{(\mu, \nu)}\left(G_{\nu}(z)\right),
\end{aligned}
$$

where $G_{\nu}(z)$ is the Cauchy transform of the measure $\nu$.

We are interested in transformations $T$ of probability measures such that for $\mu, \nu$ if we write

$$
(\xi, \eta)=(\mu, T \mu) \square(\nu, T \nu)
$$

then

$$
\eta=T \xi
$$

For such a transformation we are able to define a new convolution $\boxplus_{T}$ :

$$
\mu \boxplus_{T} \nu=(\mu, T \mu) \Subset(\nu, T \nu),
$$

and this convolution is associative. A problem of Bożejko was to find all transformations $T$ satisfying the above property. A long known example was the $t$-deformation [BW1, BW2, W] and its generalization, the $(a, b)$-deformation [KY]. Recently, Oravecz gave several new examples [O1, O2]. In this paper we study some new examples.

In section 3 we define a new deformation for which the relation (1) holds. The idea is similar to the $t$-deformation, see $[\mathrm{BW} 1, \mathrm{BW} 2, \mathrm{~W}]$ and its generalization, see [KY]. We deform the Cauchy transform of a probability measure $\mu$ by adding to its reciprocal some fixed proportion of the second free cumulant of the measure $\mu$. By the Nevanlinna theorem, the function obtained in such a way is the reciprocal of the Cauchy transform of some probability measure, which we denote by $V_{a} \mu$, that is

$$
\frac{1}{G_{V_{a} \mu}(z)}=\frac{1}{G_{\mu}(z)}+a r_{\mu}(2) .
$$

This deformation does not commute with dilation of measures. This means that the central limit theorem, proven in section 4, cannot be obtained as a direct application of the theorem for the conditionally free case. However, the central limit measure turns out to be the standard Wigner law.

In section 5 we calculate a Poisson type limit theorem and give an explicit formula for the moments of its limiting measure. A particular feature of this measure is that it puts some weight on the negative half line.

In section 6 we define another family of deformations, which also depend on the second free cumulant of the measure $\mu$. Namely, we take an arbitrary compactly supported $\boxplus$-infinitely divisible measure $\varphi$ and define a new associative convolution by applying the conditionally free convolution to pairs $\left(\mu, \varphi_{t r_{\mu}(2)}\right)$ for a fixed $t>0$. We prove the central limit theorem and Poisson type limit theorem for an arbitrary $\boxplus$-infinitely divisible measure $\varphi$. Since the dilation of measures does not commute with this deformation, namely

$$
\mathcal{D}_{\lambda} \varphi_{t r_{\mu}(2)} \neq \varphi_{t r_{\mathcal{D}_{\lambda} \mu}(2)},
$$

we cannot apply the general conditionally free limit theorems of [BLS].

We calculate the Gaussian and Poisson measures explicitly for the two particular cases when $\varphi$ is the free Poisson law $\pi_{\lambda}$ and the Wigner law $\omega$. Surprisingly, the central limit measure for the Poisson case, is not symmetric. We also give the respective Jacobi-Szegö coefficients of the measures.

\section{Preliminaries}

The Cauchy transform Let $\mu$ be a probability measure. We then denote by $G_{\mu}(z)$ the Cauchy transform of the measure $\mu$, for $z \in \mathbb{C}^{+}$, defined as follows

$$
G_{\mu}(z)=\int_{-\infty}^{+\infty} \frac{d \mu(x)}{z-x}
$$


Remark 1 The Cauchy transform $G_{\mu}(z)$ is analytic in the upper half plane and takes values in the lower half plane, $G_{\mu}(z): \mathbb{C}^{+} \longrightarrow \mathbb{C}^{-}$.

Remark 2 By the Nevanlinna theorem a function $F$ is the reciprocal of the Cauchy transform of some probability measure $\mu$ if and only if there exist a real number $\alpha$ and a positive measure $\rho$ such that

$$
F(z)=\alpha+z+\int \frac{1+x z}{x-z} d \rho(x)
$$

By a theorem of Stieltjes the Cauchy transform can be expressed as a continued fraction:

$$
G_{\mu}(z)=\frac{1}{z-\alpha_{1}-\frac{\lambda_{1}}{z-\alpha_{2}-\frac{\lambda_{2}}{z-\alpha_{3}-\frac{\lambda_{3}}{\ddots}}} .}
$$

We will denote by $G_{\mu}^{-1}(z)$ the right inverse of the Cauchy transform $G_{\mu}(z)$ with respect to composition of functions. Let us recall that for a compactly supported measure $\mu$ the function $G_{\mu}^{-1}(z)$ is well defined in some neighbourhood of zero.

We would like to remind a very useful criterion, see chapter $X I I I .6$ in [RS4] for proof:

Proposition 1 Let $\nu$ be a finite Borel measure on $\mathbb{R}$ and let $G_{\nu}(z)$ be its Cauchy transform. Let

$$
\begin{aligned}
& A_{\nu}=\left\{x: \lim _{\varepsilon \downarrow 0} G_{\nu}(x+i \varepsilon)=\infty\right\}, \\
& B_{\nu}=\left\{x: \lim _{\varepsilon \downarrow 0} G_{\nu}(x+i \varepsilon)=\Phi(x), \text { a finite number with } \Im \Phi(x) \neq 0\right\} .
\end{aligned}
$$

Then $\nu\left(\mathbb{R} \backslash\left(A_{\nu} \cup B_{\nu}\right)\right)=0, \quad \nu \uparrow A_{\nu}$ is singular relative to Lebesgue measure, and $\nu \uparrow B_{\nu}$ is absolutely continuous.

Moment-cumulant formulae Speicher [S] discovered the moment-cumulant formulae for the free convolution of Voiculescu

$$
m_{\nu}(n)=\int x^{n} d \nu(x)=\sum_{\pi \in N C(n)} r_{\nu}(\pi)=\sum_{\substack{\pi \in N C(n) \\ \pi=\left\{B_{1}, \ldots, B_{k}\right\}}} \prod_{i=1}^{k} r_{\nu}\left(\left|B_{i}\right|\right)
$$

where $\mathrm{NC}(n)$ is the set of noncrossing partitions of the set $\{1, \ldots, n\}$. Solving the above equation for $r_{\mu}(n)$ we obtain

$$
r_{\nu}(n)=m_{\nu}(n)-\sum_{\substack{\pi \in N C(n) \\ \pi \neq(1, \ldots, n)}} r_{\nu}(\pi)=m_{\nu}(n)-\sum_{\substack{\pi \in N C(n) \\ \pi \neq(1, \ldots, n)}} \prod_{i=1}^{k} r_{\nu}\left(\left|B_{i}\right|\right) .
$$

where $(1, \ldots, n)$ denotes the partition with one block containing all the points. In the case of the conditionally free cumulants of a pair $(\mu, \nu)$ Bożejko, Leinert and Speicher proved in the paper [BLS] the following relation

$$
m_{\mu}(n)=\sum_{\pi \in \mathrm{NC}(n)}\left(\prod_{\substack{B_{i} \in \pi \\ B_{i} \text {-inner }}} R_{\nu}\left(\left|B_{i}\right|\right)\right)\left(\prod_{\substack{B_{i} \in \pi \\ B_{i} \text { - outer }}} R_{(\mu, \nu)}\left(\left|B_{i}\right|\right)\right)
$$

Hence we obtain

$$
R_{(\mu, \nu)}(n)=m_{\mu}(n)-\sum_{\substack{\pi \in \mathrm{NC}(n) \\ \pi \neq(1, \ldots, n)}}\left(\prod_{\substack{B_{i} \in \pi \\ B_{i} \text { - inner }}} R_{\nu}\left(\left|B_{i}\right|\right)\right)\left(\prod_{\substack{B_{i} \in \pi \\ B_{i} \text { - outer }}} R_{(\mu, \nu)}\left(\left|B_{i}\right|\right)\right)
$$


By $[\mathrm{AB}]$ we have for a probability measure $\mu$ a relation between its moments and the Jacobi coefficients of the respective orthogonal polynomials

$$
m_{\mu}(n)=\sum_{\substack{\pi \in N C(n) \\ \pi=\left\{B_{1}, \ldots, B_{k}\right\}}} \prod_{i=1}^{k} r_{\mu}\left(\left|B_{i}\right|\right)=\sum_{\substack{\pi \in N_{1}, 2 \\ \pi=\left\{B_{1}, \ldots, B_{k}\right\}}} \prod_{\left|B_{i}\right|=2} \lambda\left(d\left(B_{i}\right)\right) \prod_{\left|B_{j}\right|=1} \alpha\left(d\left(B_{j}\right)\right),
$$

where $\mathrm{NC}_{1,2}(n)$ is the subset of noncrossing partitions consisting of partitions with blocks of size at most 2 . The sequences $\alpha_{n}, \lambda_{n}$ are the Szegö-Jacobi parameters of the recurrence formula for the orthonormal polynomials with respect to $\mu$ :

$$
\begin{aligned}
p_{0}^{\mu}(x) & =1, \quad p_{1}^{\mu}(x)=\frac{x-\alpha_{1}}{\sqrt{\lambda_{1}}}, \\
\left(x-\alpha_{n+1}\right) p_{n}^{\mu}(x) & =\sqrt{\lambda_{n+1}} p_{n+1}^{\mu}(x)+\sqrt{\lambda_{n}} p_{n-1}^{\mu}(x),
\end{aligned}
$$

and $d\left(B_{j}\right)$ denotes the depth of the block $B_{j}$ in the partition $V$, defined as 1 plus the number of blocks enveloping $B_{j}$, that is

$$
d\left(B_{j}\right)=1+\left|\left\{B_{i}: \exists r, s \in B_{i}, r<B_{j}<s\right\}\right|
$$

From this we get the following

Proposition 2

$$
r_{\mu}(1)=\alpha_{1}, \quad r_{\mu}(2)=\lambda_{1}
$$

Dilation of measures For a probability measure $\mu$ and positive number $\lambda$ we will denote by $\mathcal{D}_{\lambda} \mu$ the dilation by $\lambda$, i.e.

$$
\mathcal{D}_{\lambda} \mu(A)=\mu\left(\lambda^{-1} A\right) .
$$

Let us note, that we have the following relation between moments of a measure $\mu$ and of its dilation $\mathcal{D}_{\lambda} \mu$

$$
m_{\mathcal{D}_{\lambda} \mu}(n)=\lambda^{n} m_{\mu}(n)
$$

From the above relation we obtain

$$
r_{\mathcal{D}_{\lambda} \mu}(2)=\lambda^{2} r_{\mu}(2)
$$

\section{$3 V_{a}$-transformation}

Definition 1 For a measure $\mu$ with finite second moment and $a \in \mathbb{R}$ let us define its deformation $V_{a} \mu$ by letting

$$
\frac{1}{G_{V_{a} \mu}(z)}=\frac{1}{G_{\mu}(z)}+a r_{\mu}(2) \text {. }
$$

From the Nevanlinna theorem on the reciprocals of Cauchy transforms of measures we get that $V_{a} \mu$ is again a probability measure.

Remark 3 The $V_{a}$-transformation of a compactly supported measure can be illustrated with the use of the continued fraction representation of the Cauchy transform. Let

$$
G_{\mu}(z)=\frac{1}{z-\alpha_{1}-\frac{\lambda_{1}}{z-\alpha_{2}-\frac{\lambda_{2}}{z-\alpha_{3}-\frac{\lambda_{3}}{z-\alpha_{4}-\ddots}}}},
$$


then

$$
G_{V_{a} \mu}(z)=\frac{1}{z-\alpha_{1}+a \lambda_{1}-\frac{\lambda_{1}}{z-\alpha_{2}-\frac{\lambda_{2}}{z-\alpha_{3}-\frac{\lambda_{3}}{z-\alpha_{4}-\ddots}}} .} .
$$

This means that the $V_{a}$-transformation adds the amount a $\lambda_{1}$ to the coefficient $\alpha_{1}$.

We also have the following

Proposition 3 The $V_{a}$-transformation leaves the $\lambda_{1}$ coefficient (and thus the second cumulant) unchanged:

$$
r_{V_{a} \mu}(2)=r_{\mu}(2)
$$

This follows from the definition of $V_{a}$ and from Proposition 2.

Example 1 For the Dirac measure $\delta_{b}$ we have $V_{a} \delta_{b}=\delta_{b}$. Moreover, the Dirac measure is the only invariant measure of the $V_{a}$-transformation. This follows from the fact that the second free cumulant of an arbitrary measure $\mu \neq \delta_{b}$ is nonzero.

Example 2 We compute the $V_{a}$-transformation of a probability measure which is supported in two points. Let

$$
\mu=p \delta_{x}+q \delta_{y}, \quad x<y, \quad p, q \geq 0, \quad p+q=1 .
$$

By definition

$$
G_{V_{a} \mu}(z)=\frac{1}{\frac{1}{G_{\mu}(z)}+a r_{\mu}(2)}
$$

and because of

$$
\begin{aligned}
G_{\mu}(z) & =\frac{p}{z-x}+\frac{q}{z-y}=\frac{z-p y-q x}{z^{2}-(x+y) z+x y} \\
r_{\mu}(2) & =p q(x-y)^{2}
\end{aligned}
$$

we can obtain

$$
G_{V_{a} \mu}(z)=\frac{1}{\frac{z^{2}-(x+y) z+x y}{z-p y-q x}+a p q(x-y)^{2}}=\frac{R_{1}(z)}{R_{2}(z)}
$$

where $R_{1}, R_{2}$ are polynomials degrees of which are equal to 1,2 respectively. To be specific

$$
\frac{R_{1}(z)}{R_{2}(z)}=\frac{z-q x-p y}{z^{2}-\left(x+y-a p q(x-y)^{2}\right) z+x y-a p q(x-y)^{2}(q x+p y)} .
$$

This means, that $V_{a} \mu$ is again a two point measure

$$
V_{a} \mu=P \delta_{A}+Q \delta_{B}
$$

where $A, B$ are the zeros of the polynomial $R_{2}(z)$, that is

$$
\begin{aligned}
& A=\frac{\left(x+y-a p q(x-y)^{2}\right)-\sqrt{\left(x+y-a p q(x-y)^{2}\right)^{2}-4\left(x y-a p q(x-y)^{2}(q x+p y)\right)}}{2}, \\
& B=\frac{\left(x+y-a p q(x-y)^{2}\right)+\sqrt{\left(x+y-a p q(x-y)^{2}\right)^{2}-4\left(x y-a p q(x-y)^{2}(q x+p y)\right)}}{2} .
\end{aligned}
$$


We know that

$$
\frac{P}{z-A}+\frac{Q}{z-B}=\frac{R_{1}(z)}{R_{2}(z)}
$$

which gives

$$
\begin{aligned}
& P=\frac{1}{2}-\frac{(q-p)(x-y)+a p q(x-y)^{2}}{2 \sqrt{\left(x+y-a p q(x-y)^{2}\right)^{2}-4\left(x y-a p q(x-y)^{2}(q x+p y)\right)}}, \\
& Q=\frac{1}{2}+\frac{(q-p)(x-y)+a p q(x-y)^{2}}{2 \sqrt{\left(x+y-a p q(x-y)^{2}\right)^{2}-4\left(x y-a p q(x-y)^{2}(q x+p y)\right)}} .
\end{aligned}
$$

Remark $4 V_{a}$ is an additive group:

$$
V_{a}\left(V_{b}(\mu)\right)=V_{a+b}(\mu), \quad V_{0}(\mu)=\mu .
$$

The inverse of the transformation is $V_{a}^{-1}=V_{-a}$.

Proposition 4 We have

$$
\frac{1}{G_{V_{a}\left(\mathcal{D}_{\lambda} \mu\right)}(z)}=\frac{1}{G_{\mathcal{D}_{\lambda}\left(V_{a} \mu\right)}(z)}+a\left(\lambda^{2}-\lambda\right) r_{\mu}(2)
$$

which means that dilations of measures different from $\delta_{b}, b \in \mathbb{R}$ do not commute with $V_{a}$ for $a \neq 0$ :

$$
\mathcal{D}_{\lambda}\left(V_{a} \mu\right) \neq V_{a}\left(\mathcal{D}_{\lambda} \mu\right) .
$$

Proof: By definition of dilation and (9), (10) for $\lambda>0$ we obtain

$$
G_{\mathcal{D}_{\lambda} \mu}(z)=\int_{-\infty}^{\infty} \frac{1}{z-x} d\left(\mathcal{D}_{\lambda} \mu\right)(x)=\int_{-\infty}^{\infty} \frac{1}{z-\lambda x} d \mu(x)=\frac{1}{\lambda} G_{\mu}\left(\frac{z}{\lambda}\right),
$$

hence

$$
\frac{1}{G_{V_{a}\left(\mathcal{D}_{\lambda} \mu\right)}(z)}=\frac{1}{G_{\mathcal{D}_{\lambda} \mu}(z)}+a r_{\mathcal{D}_{\lambda} \mu}(2)=\frac{\lambda}{G_{\mu}\left(\frac{z}{\lambda}\right)}+a \lambda^{2} r_{\mu}(2) .
$$

On the other hand

$$
\frac{1}{G_{\mathcal{D}_{\lambda}\left(V_{a} \mu\right)}(z)}=\frac{\lambda}{G_{V_{a} \mu}\left(\frac{z}{\lambda}\right)}=\frac{\lambda}{G_{\mu}\left(\frac{z}{\lambda}\right)}+a \lambda r_{\mu}(2)
$$

and therefore

$$
\frac{1}{G_{V_{a}\left(\mathcal{D}_{\lambda} \mu\right)}(z)}=\frac{1}{G_{\mathcal{D}_{\lambda}\left(V_{a} \mu\right)}(z)}+a\left(\lambda^{2}-\lambda\right) r_{\mu}(2)
$$

Proposition 5 For a probability measure $\mu$ the $V_{a}$-transformation is continuous in the $*$-weak topology of measures: if $\mu_{n} \rightarrow \mu$ then $V_{a} \mu_{n} \rightarrow V_{a} \mu$. Moreover $V_{a} \mu \rightarrow \mu$ in the $*$-weak topology for $a \rightarrow 0$. This follows from the convergence of the respective Cauchy transforms.

We have the following recurrence relation between the moments of the original measure $\mu$ and of its deformation $V_{a} \mu$ :

Proposition 6 For any positive integer $n$ we have

$$
m_{V_{a} \mu}(n)=m_{\mu}(n)-a r_{\mu}(2) \sum_{k=0}^{n-1} m_{\mu}(k) m_{V_{a} \mu}(n-k-1)
$$


Proof: From the relation between the Cauchy transform of a measure $\mu$ and the generating function of its moments $M_{\mu}(z)=\sum_{n=0}^{\infty} m_{\mu}(n) z^{n}$, namely

$$
G_{\mu}(z)=\frac{1}{z} M_{\mu}\left(\frac{1}{z}\right)
$$

we get

$$
M_{V_{a} \mu}(z)=M_{\mu}(z)-a z r_{\mu}(2) M_{V_{a} \mu}(z) M_{\mu}(z) .
$$

Using the Leibniz formula for differentiation and evaluating the result at $z=0$ we obtain the desired formula.

\section{$4 V_{a}$-transformation of convolution and cumulants}

There are two ways of using a deformation of measures to define a deformation of free convolution that we are interested in. The first was mentioned in the introduction and uses the conditionally free convolution:

$$
\left(\mu \boxplus_{V_{a}} \nu, V_{a} \mu \boxplus V_{a} \nu\right)=\left(\mu, V_{a} \mu\right) \varpi\left(\nu, V_{a} \nu\right),
$$

where $\mu$ and $\nu$ are compactly supported. The second one consists in the following

Definition 2 The $V_{a}$-deformed free convolution $\square$ is defined by

$$
\mu \square \nu=V_{-a}\left(V_{a} \mu \boxplus V_{a} \nu\right)
$$

where $\mu$ and $\nu$ have finite second moments.

Remark 5 The free convolution $\boxplus$ in Definition 2 can be replaced by any associative convolution $\oplus$ (for instance by the classical convolution), producing another associative convolution $\oplus_{a}$. It is going to be the subject of a forthcoming paper.

Theorem 1 Let $\mu$ and $\nu$ be probability measures with compact support. Write $(\xi, \eta)=\left(\mu, V_{a} \mu\right) \square\left(\nu, V_{a} \nu\right)$. Then

$$
\xi=V_{-a} \eta=V_{-a}\left(V_{a} \mu \boxplus V_{a} \nu\right),
$$

that is

$$
(\xi, \eta)=\left(\xi, V_{a} \xi\right)
$$

This means that the convolutions $\boxplus_{V_{a}}$ and $\square$ are the same for measure with compact support.

Proof: By equations (3) and (4), to a pair $\left(\mu, V_{a} \mu\right)$ there correspond the two transforms with respect to the conditionally free convolution

$$
\begin{aligned}
G_{\mu}(z) & =\frac{1}{z-R_{\left(\mu, V_{a} \mu\right)}\left(G_{V_{a} \mu}(z)\right)} \\
G_{V_{a} \mu}(z) & =\frac{1}{z-R_{V_{a} \mu}\left(G_{V_{a} \mu}(z)\right)} .
\end{aligned}
$$

Thus by (11) we get

$$
R_{\left(\mu, V_{a} \mu\right)}(z)=R_{V_{a} \mu}(z)+a r_{\mu}(2) .
$$

To the pair $(\xi, \eta)$ there correspond another two series of transforms, being the sums of the respective transforms for $\left(\mu, V_{a} \mu\right)$ and $\left(\nu, V_{a} \nu\right)$ :

$$
\begin{aligned}
R_{\eta}(z) & =R_{V_{a} \mu \boxplus V_{a} \nu}(z)=R_{V_{a} \mu}(z)+R_{V_{a} \nu}(z) \\
R_{(\xi, \eta)}(z) & =R_{\left(\mu, V_{a} \mu\right)}(z)+R_{\left(\nu, V_{a} \nu\right)}(z)=R_{V_{a} \mu}(z)+a r_{\mu}(2)+R_{V_{a} \nu}(z)+a r_{\nu}(2) \\
& =R_{\eta}(z)+a\left(r_{\mu}(2)+r_{\nu}(2)\right)=R_{\eta}(z)+a\left(r_{V_{a} \mu}(2)+r_{V_{a} \nu}(2)\right)=R_{\eta}(z)+a r_{\eta}(2),
\end{aligned}
$$

where the last transition follows from Proposition 3. Thus by (15) and (16) we have

$$
\frac{1}{G_{\xi}(z)}=\frac{1}{G_{\eta}(z)}-a r_{\eta}(2)
$$

hence $\xi=V_{-a} \eta$. 
Remark 6 In the sequel we shall always assume that the measures under consideration have compact support.

Proposition 7 For any $a \in \mathbb{R}$ and any positive integer $n$ define

$$
R_{\mu}^{a}(n):=R_{V_{a} \mu}(n) .
$$

Then

$$
R_{\mu \square \nu}^{a}(n)=R_{\mu}^{a}(n)+R_{\nu}^{a}(n) .
$$

To discuss the moment-cumulant formula for the $V_{a}$-convolution we shall use the symbol outs $(\pi)$ which is the set of outer singletons of the non-crossing partition $\pi$.

Theorem 2 The moment-cumulant formula for the $a$-convolution is given by

$$
m_{\mu}(n)=\sum_{\pi \in \mathrm{NC}(n)}\left(R_{\mu}^{a}(1)+a R_{\mu}^{a}(2)\right)^{\# \text { outs }(\pi)} \prod_{\substack{B \in \pi \\ B \notin \text { outs }(\pi)}} R_{\mu}^{a}(|B|) .
$$

Proof: By definition we have

$$
\frac{1}{G_{V_{a} \mu}(z)}=\frac{1}{G_{\mu}(z)}+a r_{\mu}(2)
$$

Hence substituting $G_{V_{a} \mu}^{-1}(z)$ into $z$ we obtain

$$
\frac{1}{z}=\frac{1}{G_{\mu}\left(G_{V_{a} \mu}^{-1}(z)\right)}+a r_{\mu}(2) .
$$

Because the relations (15), (16) are equivalent to

$$
\begin{aligned}
\frac{1}{z} & =G_{V_{a} \mu}^{-1}(z)-R_{\mu}^{a}(z) \\
\frac{1}{G_{\mu}\left(G_{V_{a} \mu}^{-1}(z)\right)} & =G_{V_{a} \mu}^{-1}(z)-R_{\left(\mu, V_{a} \mu\right)}(z)
\end{aligned}
$$

by (17)

$$
G_{V_{a} \mu}^{-1}(z)-R_{\mu}^{a}(z)=G_{V_{a} \mu}^{-1}(z)-R_{\left(\mu, V_{a} \mu\right)}(z)+a r_{\mu}(2)
$$

and we get

$$
R_{\mu}^{a}(z)=R_{\left(\mu, V_{a} \mu\right)}(z)-a r_{\mu}(2) .
$$

Writing the $R$ transforms on both sides of the above equation as power series we obtain

$$
\sum_{n \geq 1} R_{\mu}^{a}(n) z^{n-1}=\sum_{n \geq 1} R_{\left(\mu, V_{a} \mu\right)}(n) z^{n-1}-a r_{\mu}(2),
$$

since

$$
r_{\mu}(1)=R_{\left(\mu, V_{a} \mu\right)}(1)
$$

which implies

$$
\begin{aligned}
& R_{\mu}^{a}(1)=r_{\mu}(1)-a r_{\mu}(2), \\
& R_{\mu}^{a}(n)=R_{\left(\mu, V_{a} \mu\right)}(n), \quad n \geq 2 .
\end{aligned}
$$

Thus, we obtain from (6) and Proposition 3

$$
m_{\mu}(n)=\sum_{\pi \in \mathrm{NC}(n)}\left(R_{\mu}^{a}(1)+a R_{\mu}^{a}(2)\right)^{\# \operatorname{outs}(\pi)} \prod_{\substack{B \in \pi \\ B \notin \text { outs }(\pi)}} R_{\mu}^{a}(|B|) .
$$

We would like now to establish a central limit theorem for the $a$ convolution. First we will prove some technical lemmas. 
Lemma 1 Let $\mu$ be a compactly supported probability measure on the real line. Then

$$
m_{V_{a} \mathcal{D}_{\lambda} \mu}(n)=\lambda^{n} m_{\mu}(n)+o\left(\lambda^{n}\right)
$$

Proof: Using the relation between moments of measure and of its dilation (9) and Proposition 6 we obtain

$$
\begin{aligned}
m_{V_{a} \mathcal{D}_{\lambda} \mu}(n) & =m_{\mathcal{D}_{\lambda} \mu}(n)-a r_{\mathcal{D}_{\lambda} \mu}(2) \sum_{k=0}^{n-1} m_{\mathcal{D}_{\lambda} \mu}(k) m_{V_{a} \mathcal{D}_{\lambda} \mu}(n-k-1) \\
& =\lambda^{n} m_{\mu}(n)-a \lambda^{2} r_{\mu}(2) \sum_{k=0}^{n-1} \lambda^{k} m_{\mu}(k) m_{V_{a} \mathcal{D}_{\lambda} \mu}(n-k-1)
\end{aligned}
$$

In particular for $\lambda \rightarrow 0$ and for $n=1$ we have

$$
m_{V_{a} \mathcal{D}_{\lambda} \mu}(1)=\lambda m_{\mu}(1)-a \lambda^{2} r_{\mu}(2)
$$

Hence by induction

$$
\begin{aligned}
m_{V_{a} \mathcal{D}_{\lambda} \mu}(n+1) & =\lambda^{n+1} m_{\mu}(n+1)-a \lambda^{2} r_{\mu}(2) \sum_{k=0}^{n} \lambda^{k} m_{\mu}(k) m_{V_{a} \mathcal{D}_{\lambda} \mu}(n-k) \\
& =\lambda^{n+1} m_{\mu}(n+1)-a \lambda^{2} r_{\mu}(2) \sum_{k=0}^{n} \lambda^{k} m_{\mu}(k)\left(\lambda^{n-k} m_{\mu}(n-k)+o\left(\lambda^{n-k+1}\right)\right) \\
& =\lambda^{n+1} m_{\mu}(n+1)-o\left(\lambda^{n+2}\right) a r_{\mu}(2) \sum_{k=0}^{n} m_{\mu}(k)\left(m_{\mu}(n-k)+o(\lambda)\right) \\
& =\lambda^{n+1} m_{\mu}(n+1)-o\left(\lambda^{n+2}\right) a r_{\mu}(2) \sum_{k=0}^{n} m_{\mu}(k) m_{\mu}(n-k)-o\left(\lambda^{n+3}\right) a r_{\mu}(2) \sum_{k=0}^{n} m_{\mu}(k) \\
& =\lambda^{n+1} m_{\mu}(n+1)+o\left(\lambda^{n+3}\right)
\end{aligned}
$$

The last inequality follows from the fact that for compactly supported measure $\mu$ there exists a constant $c$ such that

$$
\left|m_{\mu}(k)\right| \leq c^{k}
$$

hence

$$
\begin{array}{r}
\sum_{k=0}^{n} m_{\mu}(k) \leq c \frac{1-c^{n+1}}{1-c} \\
\sum_{k=0}^{n} m_{\mu}(k) m_{\mu}(n-k) \leq(n+1) c^{j}
\end{array}
$$

Thus we obtain

$$
m_{V_{a} \mathcal{D}_{\lambda} \mu}(n+1)=\lambda^{n+1} m_{\mu}(n+1)+o\left(\lambda^{n+1}\right) .
$$

Lemma 2 Let $\mu$ be a compactly supported probability measure on the real line with mean zero and variance equal to 1. Then

$$
\begin{aligned}
& R_{\mathcal{D}_{\lambda} \mu}^{a}(1)=r_{V_{a} \mathcal{D}_{\lambda} \mu}(1)=-a \lambda^{2}, \\
& R_{\mathcal{D}_{\lambda} \mu}^{a}(2)=r_{V_{a} \mathcal{D}_{\lambda} \mu}(2)=\lambda^{2}, \\
& R_{\mathcal{D}_{\lambda} \mu}^{a}(k)=r_{V_{a} \mathcal{D}_{\lambda} \mu}(k)=o\left(\lambda^{2}\right) \quad \text { for } k \geq 3 .
\end{aligned}
$$

Proof: Let us observe that for a measure $\mu$ with mean zero and variance equal to 1 the measure $\mathcal{D}_{\lambda} \mu$ has the first moment $m_{\mathcal{D}_{\lambda} \mu}(1)=0$ and the second cumulant $r_{\mathcal{D}_{\lambda} \mu}(2)=\lambda^{2}$. The measure $V_{a} \mathcal{D}_{\lambda} \mu$ has the first moment $m_{V_{a} \mathcal{D}_{\lambda} \mu}(1)=-a \lambda^{2}$ and the second cumulant $r_{V_{a} \mathcal{D}_{\lambda} \mu}(2)=\lambda^{2}$. 
Because of the moment-cumulant formulae for the free convolution (5) for the $V_{a}$-deformation of dilation of measures we have

$$
\begin{aligned}
& r_{V_{a} \mathcal{D}_{\lambda} \mu}(1)=-a \lambda^{2}, \\
& r_{V_{a} \mathcal{D}_{\lambda} \mu}(2)=\lambda^{2} .
\end{aligned}
$$

Moreover, for $k=3$ by the moment-cumulant formula (5)

$$
r_{V_{a} \mathcal{D}_{\lambda} \mu}(3)=m_{V_{a} \mathcal{D}_{\lambda} \mu}(3)-3 r_{V_{a} \mathcal{D}_{\lambda} \mu}(2) r_{V_{a} \mathcal{D}_{\lambda} \mu}(1)-r_{V_{a} \mathcal{D}_{\lambda} \mu}(1)^{3}
$$

by Lemma 1 we have

$$
r_{V_{a} \mathcal{D}_{\lambda} \mu}(3)=\lambda^{3} m_{\mu}(3)+o\left(\lambda^{3}\right)+3 a \lambda^{4}+a^{3} \lambda^{6},
$$

and by induction: if in $\pi$ there exists $B$ such that $|B| \geq 3$, then $r_{V_{a} \mathcal{D}_{\lambda} \mu}(\pi)=o\left(\lambda^{2}\right)$. If not, there must be at least two blocks, $\left|B_{1}\right|+\left|B_{2}\right| \geq 2$, hence

$$
\prod_{B_{i} \in \pi} r_{V_{a} \mathcal{D}_{\lambda} \mu}\left(B_{i}\right)=o\left(\lambda^{3}\right)
$$

Theorem 3 (Central limit theorem) Let $\mu$ be a compactly supported probability measure on the real line with mean zero and variance equal to 1 . Then the sequence

$$
\mathcal{D}_{1 / \sqrt{N}} \mu \square \ldots a \mathcal{D}_{1 / \sqrt{N}} \mu
$$

is $*$-weakly convergent to the standard semicircular law $\omega$ with density

$$
d \omega(x)=\frac{1}{2 \pi} \sqrt{4-x^{2}} d x .
$$

Proof: The sequence of $n$-fold $V_{a}$-convolution of the measure $\mu$ is of the form

$$
\mu_{N}=\mathcal{D}_{1 / \sqrt{N}} \mu \square \ldots \square \mathcal{D}_{1 / \sqrt{N}} \mu=V_{-a}\left(V_{a} \mathcal{D}_{1 / \sqrt{N}} \mu \boxplus \ldots \boxplus V_{a} \mathcal{D}_{1 / \sqrt{N}} \mu\right)
$$

Taking $\lambda=\frac{1}{\sqrt{N}}$ let us denote

$$
\nu_{N}=\underbrace{V_{a} \mathcal{D}_{1 / \sqrt{N}} \mu \boxplus \cdots \boxplus V_{a} \mathcal{D}_{1 / \sqrt{N}} \mu}_{N \text { times }}
$$

Then $\mu_{N}=V_{-a} \nu_{N}$ and by definition

$$
\begin{aligned}
R_{\nu}^{a}(z) & =R_{V_{a} \nu}(z) \\
R_{\nu_{N}}(z) & =R_{V_{a} \mu_{N}}(z)=R_{\mu_{N}}^{a}(z)
\end{aligned}
$$

and writing $R_{\nu}(a) z$ as a power series we obtain

$$
R_{\nu}^{a}(z)=\sum_{n=1}^{\infty} r_{V_{a} \nu}(n) z^{n-1}
$$

Hence

$$
R_{\nu_{N}}(z)=N R_{V_{a} \mathcal{D}_{1 / \sqrt{N}} \mu}(z)=N R_{\mathcal{D}_{1 / \sqrt{N}} \mu}^{a}(z)
$$

and by Lemma 2

$$
\begin{aligned}
& r_{\nu_{N}}(1)=-N \cdot \frac{a}{N}=-a \\
& r_{\nu_{N}}(2)=N \cdot \frac{1}{N}=1 \\
& r_{\nu_{N}}(k)=N \cdot o\left(\frac{1}{N}\right) \rightarrow_{N \rightarrow \infty} 0
\end{aligned}
$$


and therefore

$$
R_{\nu_{N}}(z) \rightarrow R_{\omega_{-a}}(z)=-a+z,
$$

and $\omega_{-a}$ is the semicircular law with the first moment $-a$ and variance 1. Moreover

$$
R_{\mu_{N}}(z)=R_{\nu_{N}}^{-a}(z) \rightarrow R_{\omega_{-a}}^{-a}(z)=R_{V_{-a} \omega_{-a}}(z) .
$$

Because of

$$
G_{\omega_{-a}}(z)=\frac{1}{z+a-\frac{1}{z-\frac{1}{z-\frac{1}{\ddots}}}}
$$

we have

$$
G_{V_{-a} \omega_{-a}}(z)=\frac{1}{z-\frac{1}{z-\frac{1}{z-\frac{1}{\ddots}}}}
$$

Therefore

$$
R_{\mu_{N}}(z) \rightarrow R_{\omega}(z)=z
$$

and

$$
\mathcal{D}_{1 / \sqrt{N}} \mu \square \ldots \square \mathcal{D}_{1 / \sqrt{N}} \mu=V_{-a}\left(V_{a} \mathcal{D}_{1 / \sqrt{N}} \mu \boxplus \ldots \boxplus V_{a} \mathcal{D}_{1 / \sqrt{N}} \mu\right) \rightarrow \omega,
$$

where $\omega$ is the standard semicircular law with mean 0 and variance 1.

\section{Poisson type limit theorem}

In this section we will study a Poisson limit theorem for the $\square$ convolution.

Theorem 4 Let $\lambda>0$. Consider the sequence of measures

$$
\mu_{N}=\left(1-\frac{\lambda}{N}\right) \delta_{0}+\frac{\lambda}{N} \delta_{1}, \quad N \geq 1
$$

The sequence

$$
\lim _{N \rightarrow \infty} \underbrace{\mu_{N} \square \cdots \square \mu_{N}}_{N}
$$

is convergent in the weak $*$-topology to a measure $p_{\lambda}$ which we call the $V_{a}$-free Poisson law. The measure $p_{\lambda}$ is of the form $p_{\lambda}=\tilde{p}_{\lambda}+\hat{p}_{\lambda}$, where $\tilde{p}_{\lambda}$ is the continuous part with density

$$
d \tilde{p}_{\lambda}(x)=\frac{\sqrt{4 \lambda-(x+a \lambda-\lambda-1)^{2}}}{2 \pi\left((1-a \lambda) x+a \lambda^{2}\right)} \chi_{[\lambda+1-a \lambda-2 \sqrt{\lambda}, \lambda+1-a \lambda+2 \sqrt{\lambda}]} d x
$$

and $\hat{p}_{\lambda}$ is the discrete part

$$
\hat{p}_{\lambda}(x)=\max \left(0,1-\frac{\lambda}{(a \lambda-1)^{2}}\right) \delta_{\frac{a \lambda^{2}}{a \lambda-1}}
$$


Proof: Set $p_{N}=\frac{\lambda}{N}$ and consider the measures

$$
\mu_{N}=\left(1-p_{N}\right) \delta_{0}+p_{N} \delta_{1}, \quad N \geq 1
$$

A straightforward calculation shows that

$$
\begin{aligned}
G_{\mu_{N}}(z) & =\frac{z-\left(1-p_{N}\right)}{z(z-1)} \\
G_{V_{a} \mu_{N}}(z) & =\frac{z-1+p_{N}}{(z-1) z+a p_{N}\left(1-p_{N}\right)\left(z-1+p_{N}\right)} .
\end{aligned}
$$

Thus by the definition of the conditionally free $R$-transform we get

$$
\begin{gathered}
R_{\left(\mu_{N}, V_{a} \mu_{N}\right)}\left(G_{V_{a}\left(\mu_{N}\right)}(z)\right)=z-\frac{1}{G_{\mu_{N}}(z)} \\
R_{\left(\mu_{N}, V_{a} \mu_{N}\right)}\left(\frac{z-1+p_{N}}{(z-1) z+a p_{N}\left(1-p_{N}\right)\left(z-1+p_{N}\right)}\right)=z-\frac{z(z-1)}{z-\left(1-p_{N}\right)}=\frac{z p_{N}}{z-1+p_{N}},
\end{gathered}
$$

so

$$
R_{\left(\mu_{N}, V_{a} \mu_{N}\right)}(z)=\frac{p_{N} w}{w-1+p_{N}}
$$

where

$$
w=\frac{1+z-a z p_{N}+a z p_{N}^{2}+\sqrt{\left(1+z-a z p_{N}+a z p_{N}^{2}\right)^{2}-4 z\left(1-p_{N}-a z p_{N}\left(1-2 p_{N}+p_{N}^{2}\right)\right)}}{2 z}
$$

and the square root has branch-cut discontinuity on the negative half-axis.

For $N \rightarrow \infty$ the function $N \cdot R_{\left(\mu_{N}, V_{a} \mu_{N}\right)}(z)$ tends uniformly to

$$
R_{\left(p_{\lambda}, V_{a} p_{\lambda}\right)}(z)=\frac{\lambda}{1-z}
$$

By (15) we obtain the following Cauchy transform of the limiting measure

$$
G_{p_{\lambda}}(z)=\frac{1}{z-\frac{\lambda}{1-G_{V_{a} p_{\lambda}}(z)}}
$$

and because of

$$
\begin{aligned}
r_{p_{\lambda}}(2) & =R_{\left(p_{\lambda}, V_{a} p_{\lambda}\right)}(2)=\lambda \\
\frac{1}{G_{p_{\lambda}}(z)} & =\frac{1}{G_{V_{a} p_{\lambda}(z)}}-a r_{p_{\lambda}}(2),
\end{aligned}
$$

we obtain from (20)

$$
\begin{array}{r}
\frac{1}{G_{V_{a} p_{\lambda}}(z)}-a \lambda=z-\frac{\lambda}{1-G_{V_{a} p_{\lambda}}(z)}, \\
(a \lambda+z) G_{V_{a} p_{\lambda}}(z)^{2}+(\lambda-z-1-a \lambda) G_{V_{a} p_{\lambda}}(z)+1=0 .
\end{array}
$$

Hence

$$
G_{V_{a} p_{\lambda}}(z)=\frac{z+1+a \lambda-\lambda-\sqrt{(z+1+a \lambda-\lambda)^{2}-4(z+a \lambda)}}{2(z+a \lambda)}
$$

where the branch of the square root is chosen so that the Cauchy transform is continuous for $z \in \mathbb{C}^{+}$, which means that for negative $x$ we have

$$
\sqrt{x+i \epsilon} \longrightarrow \epsilon \rightarrow 0=i \sqrt{-x}
$$


whereas for positive $x$ we have for positive $\epsilon$ the limit

$$
\sqrt{x+i \epsilon} \longrightarrow \epsilon \rightarrow 0^{+}=\sqrt{x}
$$

and for negative $\epsilon$ the limit

$$
\sqrt{x+i \epsilon} \longrightarrow \epsilon_{\epsilon \rightarrow 0^{-}}=-\sqrt{x}
$$

Calculation of the explicit form of the measure $V_{a} p_{\lambda}$ can now be done by calculating $G_{p_{\lambda}}(z)$, and using the Stieltjes inversion formula $[\mathrm{Ak}]$. We can get it faster by using the continued fraction representation of Cauchy transforms and by a result by Saitoh and Yoshida [SY]. We have

$$
G_{V_{a} p_{\lambda}}(z)=\frac{z+a \lambda+1-\lambda-\sqrt{(z+a \lambda-1-\lambda)^{2}-4 \lambda}}{2(z+a \lambda)} .
$$

which can be written as

$$
G_{V_{a} p_{\lambda}}(z)=\frac{1}{\frac{z+a \lambda+1-\lambda}{2}+\sqrt{\left(\frac{z+a \lambda-1-\lambda}{2}\right)^{2}-\lambda}} .
$$

Using the well-known formula

$$
\sqrt{a^{2}+b}=a+\frac{b}{2 a+\frac{b}{2 a+\frac{b}{2 a+\frac{b}{\ddots}}}}
$$

we get

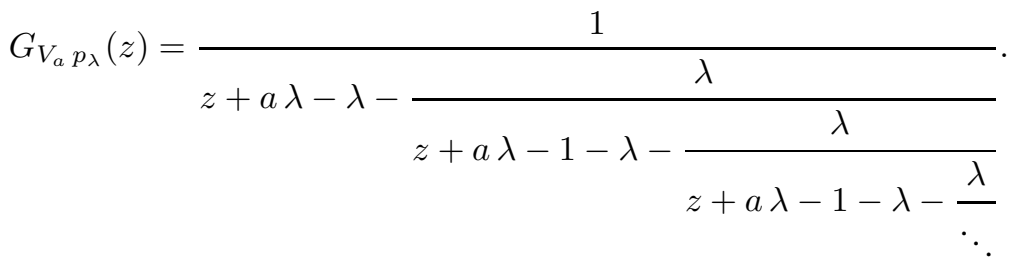

Because of $r_{p_{\lambda}}(2)=r_{V_{a} p_{\lambda}}(2)=\lambda$ we obtain

$$
G_{p_{\lambda}}(z)=\frac{1}{z-\lambda-\frac{\lambda}{z+a \lambda-1-\lambda-\frac{\lambda}{z+a \lambda-1-\lambda-\frac{\lambda}{\ddots}}} .}
$$

Using a theorem of Saitoh and Yoshida, see [SY], we obtain the unique probability measure $p_{\lambda}=\tilde{p}_{\lambda}+\hat{p}_{\lambda}$ ortogonalizing the above system of polynomials, where $\tilde{p}_{\lambda}$ is the absolutely continuous part of the measure $p_{\lambda}$ and $\hat{p}_{\lambda}$ denotes the discrete part of this measure. In our case let us denote

$$
f(x)=(1-a \lambda)(x+a \lambda-\lambda-1)+(1-a \lambda)^{2}+\lambda=(1-a \lambda) x+a \lambda^{2} .
$$

Thus we get

$$
d \tilde{p}_{\lambda}(x)=\frac{\sqrt{4 \lambda-(x+a \lambda-\lambda-1)^{2}}}{2 \pi f(x)} \chi_{[\lambda+1-a \lambda-2 \sqrt{\lambda}, \lambda+1-a \lambda+2 \sqrt{\lambda}]} d x .
$$

This measure has a discrete part unless $a \lambda=1$. Since $f(x)$ has one real root $y=\lambda+\frac{\lambda}{a \lambda-1}=\frac{a \lambda^{2}}{a \lambda-1}$, this discrete part is

$$
\hat{p}_{\lambda}(x)=\max \left(0,1-\frac{\lambda}{(a \lambda-1)^{2}}\right) \delta_{\frac{a \lambda^{2}}{a \lambda-1}} .
$$


The first few moments of the Poisson distribution of parameter $\lambda$ are

$$
\begin{aligned}
& m(0)=1, \\
& m(1)=\lambda \\
& m(2)=\lambda+\lambda^{2}, \\
& m(3)=\lambda+(3-a) \lambda^{2}+\lambda^{3}, \\
& m(4)=\lambda+(6-2 a) \lambda^{2}+\left(6-4 a+a^{2}\right) \lambda^{3}+\lambda^{4}, \\
& m(5)=\lambda+(10-3 a) \lambda^{2}+\left(20-15 a+3 a^{2}\right) \lambda^{3}+\left(10-10 a+5 a^{2}-a^{3}\right) \lambda^{4}+\lambda^{5} .
\end{aligned}
$$

Moreover, we obtain

Theorem 5 Suppose $a \lambda \neq 1$. Moments of the measure $p_{\lambda}$ are given by the following formula

$$
\begin{aligned}
m_{p_{\lambda}}(n) & =\frac{\left(-a \lambda^{2}\right)^{n}}{(1-a \lambda)^{n}} \\
& +\sum_{k=0}^{n-1} \sum_{j=0}^{\lfloor k / 2\rfloor} \frac{1}{j+1}\left(\begin{array}{c}
n \\
k+1
\end{array}\right)\left(\begin{array}{c}
k \\
2 j
\end{array}\right)\left(\begin{array}{c}
2 j \\
j
\end{array}\right)(-a)^{n-k-1}\left((1-a \lambda)^{2}+\lambda\right)^{k-2 j} \lambda^{2 n-2 k+j-1}(1-a \lambda)^{2 j-n} .
\end{aligned}
$$

Proof: Integrating with respect to the continuous part of the measure $p_{\lambda}$ we obtain

$$
\begin{aligned}
m_{\tilde{p}_{\lambda}}(n) & =\frac{1}{2 \pi} \int_{\lambda+1-a \lambda-2 \sqrt{\lambda}}^{\lambda+1-a \lambda+2 \sqrt{\lambda}} t^{n} \frac{\sqrt{4 \lambda-(t+a \lambda-\lambda-1)^{2}}}{(1-a \lambda)(t+a \lambda-\lambda-1)+(1-a \lambda)^{2}+\lambda} d t \\
& =\frac{1}{2 \pi(1-a \lambda)} \int_{-2 \sqrt{\lambda}(1-a \lambda)+(1-a \lambda)^{2}+\lambda}^{2 \sqrt{\lambda}(1-a \lambda)+(1-a \lambda)^{2}+\lambda}\left(\frac{x-a \lambda^{2}}{1-a \lambda}\right)^{n} \frac{\sqrt{4 \lambda-\left(\frac{x-\lambda}{1-a \lambda}-1+a \lambda\right)^{2}}}{x} d x \\
& =\frac{1}{(1-a \lambda)^{n+1}} \sum_{k=0}^{n}\left(\begin{array}{l}
n \\
k
\end{array}\right)\left(-a \lambda^{2}\right)^{n-k} c_{k-1},
\end{aligned}
$$

where

$$
c_{k-1}=\frac{1}{2 \pi} \int_{-2 \sqrt{\lambda}(1-a \lambda)+(1-a \lambda)^{2}+\lambda}^{2 \sqrt{\lambda}(1-a \lambda)+(1-a \lambda)^{2}+\lambda} x^{k-1} \sqrt{4 \lambda-\left(\frac{x-\lambda}{1-a \lambda}-1+a \lambda\right)^{2}} d x .
$$

Integrating by substitution once again we get

$$
\begin{aligned}
c_{k} & =\frac{1}{2 \pi} \int_{-2 \sqrt{\lambda}(1-a \lambda)+(1-a \lambda)^{2}+\lambda}^{2 \sqrt{\lambda}(1-a \lambda)+(1-a \lambda)^{2}+\lambda} x^{k} \sqrt{4 \lambda-\left(\frac{x-\lambda}{1-a \lambda}-1+a \lambda\right)^{2}} d x \\
& =\frac{1}{2 \pi} \int_{-2}^{2}\left(\sqrt{\lambda}(1-a \lambda) y+(1-a \lambda)^{2}+\lambda\right)^{k} \sqrt{4 \lambda-\lambda y^{2}} \sqrt{\lambda}(1-a \lambda) d y \\
& =\frac{\lambda(1-a \lambda)}{2 \pi} \int_{-2}^{2}\left(\sqrt{\lambda}(1-a \lambda) y+(1-a \lambda)^{2}+\lambda\right)^{k} \sqrt{4-y^{2}} d y \\
& =\frac{\lambda(1-a \lambda)}{2 \pi} \sum_{l=0}^{k}\left(\begin{array}{l}
k \\
l
\end{array}\right)\left((1-a \lambda)^{2}+\lambda\right)^{k-l}(\sqrt{\lambda}(1-a \lambda))^{l} \int_{-2}^{2} y^{l} \sqrt{4-y^{2}} d y .
\end{aligned}
$$

Because of

$$
\frac{1}{2 \pi} \int_{-2}^{2} y^{l} \sqrt{4-y^{2}} d y= \begin{cases}\frac{1}{\frac{1}{2}+1}\left(\begin{array}{l}
l \\
\frac{l}{2}
\end{array}\right) & l \text { is even } \\
0 & l \text { is odd }\end{cases}
$$


we obtain

$$
\begin{aligned}
c_{k} & =\lambda(1-a \lambda) \sum_{\substack{l=0 \\
l \text { even }}}^{k}\left(\begin{array}{c}
k \\
l
\end{array}\right)\left((1-a \lambda)^{2}+\lambda\right)^{k-l}(\sqrt{\lambda}(1-a \lambda))^{l} \frac{1}{\frac{l}{2}+1}\left(\begin{array}{c}
l \\
\frac{l}{2}
\end{array}\right) \\
& =\lambda(1-a \lambda) \sum_{j=0}^{\lfloor k / 2\rfloor}\left(\begin{array}{c}
k \\
2 j
\end{array}\right)\left((1-a \lambda)^{2}+\lambda\right)^{k-2 j} \lambda^{j}(1-a \lambda)^{2 j} \frac{1}{j+1}\left(\begin{array}{c}
2 j \\
j
\end{array}\right) .
\end{aligned}
$$

For $k=0$

$$
\begin{aligned}
& c_{-1}=\frac{1}{2 \pi} \int_{-2 \sqrt{\lambda}(1-a \lambda)+(1-a \lambda)^{2}+\lambda}^{2 \sqrt{\lambda}(1-a \lambda)+(1-a \lambda)^{2}+\lambda} \frac{\sqrt{4 \lambda-\left(\frac{x-\lambda}{1-a \lambda}-1+a \lambda\right)^{2}}}{x} d x \\
& =\frac{1}{2 \pi|1-a \lambda|} \int_{-2 \sqrt{\lambda}(1-a \lambda)+(1-a \lambda)^{2}+\lambda}^{2 \sqrt{\lambda}(1-a \lambda)+(1-a \lambda)^{2}+\lambda} \frac{\sqrt{4 \lambda(1-a \lambda)^{2}-\left(x-\lambda-(1-a \lambda)^{2}\right)^{2}}}{x} d x \\
& =\frac{1}{2 \pi|1-a \lambda|} \sqrt{4 \lambda(1-a \lambda)^{2}-\left(x-\lambda-(1-a \lambda)^{2}\right)^{2}} \\
& -\frac{\lambda+(1-a \lambda)^{2}}{2 \pi|1-a \lambda|} \arcsin \frac{\left(\lambda+(1-a \lambda)^{2}\right)-x}{2|1-a \lambda| \sqrt{\lambda}} \\
& -\frac{\left|\lambda-(1-a \lambda)^{2}\right|}{2 \pi|1-a \lambda|} \arcsin \frac{\left(\lambda+(1-a \lambda)^{2}\right) x-\left(\lambda-(1-a \lambda)^{2}\right)^{2}}{2|1-a \lambda| \sqrt{\lambda} x} \mid \begin{array}{l}
2 \sqrt{\lambda}(1-a \lambda)+(1-a \lambda)^{2}+\lambda \\
-2 \sqrt{\lambda}(1-a \lambda)+(1-a \lambda)^{2}+\lambda
\end{array} \\
& =\left(-\frac{\lambda+(1-a \lambda)^{2}}{2 \pi|1-a \lambda|}+\frac{\left|\lambda-(1-a \lambda)^{2}\right|}{2 \pi|1-a \lambda|}\right)\left(\arcsin \frac{a \lambda-1}{|1-a \lambda|}-\arcsin \frac{1-a \lambda}{|1-a \lambda|}\right) \\
& =-\frac{\lambda+(1-a \lambda)^{2}}{2(a \lambda-1)}+\frac{\left|\lambda-(1-a \lambda)^{2}\right|}{2(a \lambda-1)}= \begin{cases}1-a \lambda & \text { if } \lambda \in\left[\frac{1+2 a-\sqrt{1+4 a}}{2 a^{2}}, \frac{1+2 a+\sqrt{1+4 a}}{2 a^{2}}\right] \\
\frac{\lambda}{1-a \lambda} & \text { if } \lambda \notin\left[\frac{1+2 a-\sqrt{1+4 a}}{2 a^{2}}, \frac{1+2 a+\sqrt{1+4 a}}{2 a^{2}}\right] .\end{cases}
\end{aligned}
$$

\section{Hence}

$$
\begin{aligned}
m_{\tilde{p}_{\lambda}}(n) & =\frac{\left(-a \lambda^{2}\right)^{n} c_{-1}}{(1-a \lambda)^{n+1}} \\
& +\frac{\lambda}{(1-a \lambda)^{n}} \sum_{k=1}^{n}\left(\begin{array}{l}
n \\
k
\end{array}\right)\left(-a \lambda^{2}\right)^{n-k} \sum_{j=0}^{\lfloor(k-1) / 2\rfloor}\left(\begin{array}{c}
k-1 \\
2 j
\end{array}\right)\left((1-a \lambda)^{2}+\lambda\right)^{k-1-2 j} \lambda^{j}(1-a \lambda)^{2 j} \frac{1}{j+1}\left(\begin{array}{c}
2 j \\
j
\end{array}\right) \\
& =\frac{\left(-a \lambda^{2}\right)^{n} c_{-1}}{(1-a \lambda)^{n+1}} \\
& +\sum_{k=1}^{n} \sum_{j=0}^{\lfloor(k-1) / 2\rfloor} \frac{1}{j+1}\left(\begin{array}{c}
n \\
k
\end{array}\right)\left(\begin{array}{c}
k-1 \\
2 j
\end{array}\right)\left(\begin{array}{c}
2 j \\
j
\end{array}\right)(-a)^{n-k}\left((1-a \lambda)^{2}+\lambda\right)^{k-1-2 j} \lambda^{2 n-2 k+j+1}(1-a \lambda)^{2 j-n}
\end{aligned}
$$

Because of

we obtain

$$
m_{p_{\lambda}}(n)=m_{\tilde{p}_{\lambda}}(n)+\left(\frac{-a \lambda^{2}}{1-a \lambda}\right)^{n} \max \left(0,1-\frac{\lambda}{(a \lambda-1)^{2}}\right)
$$

$$
\begin{aligned}
m_{p_{\lambda}}(n) & =\frac{\left(-a \lambda^{2}\right)^{n} c_{-1}}{(1-a \lambda)^{n+1}}+\left(\frac{-a \lambda^{2}}{1-a \lambda}\right)^{n} \max \left(0,1-\frac{\lambda}{(a \lambda-1)^{2}}\right) \\
& +\sum_{k=1}^{n} \sum_{j=0}^{\lfloor(k-1) / 2\rfloor} \frac{1}{j+1}\left(\begin{array}{c}
n \\
k
\end{array}\right)\left(\begin{array}{c}
k-1 \\
2 j
\end{array}\right)\left(\begin{array}{c}
2 j \\
j
\end{array}\right)(-a)^{n-k}\left((1-a \lambda)^{2}+\lambda\right)^{k-1-2 j} \lambda^{2 n-2 k+j+1}(1-a \lambda)^{2 j-n} \\
& =\frac{\left(-a \lambda^{2}\right)^{n}}{(1-a \lambda)^{n}} \\
& +\sum_{k=0}^{n-1} \sum_{j=0}^{\lfloor k / 2\rfloor} \frac{1}{j+1}\left(\begin{array}{c}
n \\
k+1
\end{array}\right)\left(\begin{array}{c}
k \\
2 j
\end{array}\right)\left(\begin{array}{c}
2 j \\
j
\end{array}\right)(-a)^{n-k-1}\left((1-a \lambda)^{2}+\lambda\right)^{k-2 j} \lambda^{2 n-2 k+j-1}(1-a \lambda)^{2 j-n} .
\end{aligned}
$$




\section{Deformation connected with infinitely divisible measures}

This deformation is a generalization of ideas of Oravecz [O1, O2] and of Bryc and Wesołowski [BrW]. It uses compactly supported probability measures infinitely divisible with respect to the free convolution. The main observation, due to Bercovici and Voiculescu [BV], is the following:

Theorem 6 To any compactly supported probability measure $\varphi$ infinitely divisible with respect to the free convolution there correspond a $w^{*}$-continuous $\boxplus$-semigroup $\left(\varphi_{t}\right)$ such that $\varphi_{1}=\varphi$. This means that

$$
R_{\varphi_{t}}(z)=t R_{\varphi}(z)
$$

and

$$
r_{\varphi_{t}}(n)=t \cdot r_{\varphi}(n)
$$

Now we would like to define a new deformation and convolution.

Definition 3 Let $\varphi$ be some fixed compactly supported $\boxplus$-infinitely divisible probability measure and $\mu$ any measure with compact support. Let us consider the following map $\mu \mapsto \Phi_{t}^{\varphi} \mu$ :

$$
\Phi_{t}^{\varphi} \mu=\varphi_{t r_{\mu}(2)},
$$

which depends on the second free cumulant of the measure $\mu$ and nonnegative parameter $t$.

Proposition 8 We have the following relation for the respective transforms

$$
R_{\Phi_{t}^{\varphi} \mu}(z)=R_{\varphi_{t r_{\mu}(2)}}(z)=t r_{\mu}(2) R_{\varphi}(z) .
$$

Remark 7 Let $\varphi$ be $\boxplus$-infinitely divisible with variance equal to 1. Then $\Phi_{t}^{\varphi} \mu$ is a multiplicative semigroup. Indeed, we have

$$
\Phi_{t}^{\varphi}\left(\Phi_{s}^{\varphi} \mu\right)=\Phi_{t}^{\varphi}\left(\varphi_{s r_{\mu}(2)}\right)
$$

and by

$$
r_{\varphi_{s r_{\mu}(2)}}(2)=s r_{\mu}(2) r_{\varphi}(2)=s r_{\mu}(2)
$$

hence

$$
\Phi_{t}^{\varphi}\left(\Phi_{s}^{\varphi} \mu\right)=\varphi_{s t r_{\mu}(2)}=\Phi_{t s}^{\varphi} \mu .
$$

Proposition 9 In general the dilation of measures does not commute with $\Phi_{t}^{\varphi}$.

Proof: Indeed, we have

$$
\Phi_{t}^{\varphi}\left(\mathcal{D}_{\lambda} \mu\right)=\varphi_{t \lambda^{2} r_{\mu}(2)}
$$

and on the other hand

$$
\mathcal{D}_{\lambda}\left(\Phi_{t}^{\varphi} \mu\right)=\mathcal{D}_{\lambda} \varphi_{t r_{\mu}(2)}
$$

Moreover, these measures have in general different cumulants:

$$
\begin{aligned}
r_{\Phi_{t}^{\varphi}\left(\mathcal{D}_{\lambda} \mu\right)}(n) & =t \lambda^{2} r_{\mu}(2) r_{\varphi}(n) \\
r_{\mathcal{D}_{\lambda} \Phi_{t}^{\varphi} \mu}(n) & =t \lambda^{n} r_{\mu}(2) r_{\varphi}(n) .
\end{aligned}
$$

The last equality follows from the fact, that $R_{\mathcal{D}_{\lambda} \mu}(z)=\lambda R_{\mu}(\lambda z)$. 
Proposition 10 For a probability measure $\mu$ the $\Phi_{t}^{\varphi}$-transformation is continuous in the *-weak topology of measures: if $\mu_{n} \rightarrow \mu$ then $\Phi_{t}^{\varphi} \mu_{n} \rightarrow \Phi_{t}^{\varphi} \mu$. Moreover $\Phi_{t}^{\varphi} \mu \rightarrow \delta_{0}$ in the $*$-weak topology for $t \rightarrow 0$. This follows from the convergence of the respective $R$-transforms.

Definition 4 For probability measures $\mu, \nu$ we define their $\varphi$-convolution by

$$
\mu \varphi \nu=\left(\mu, \Phi_{t}^{\varphi} \mu\right) 匹\left(\nu, \Phi_{t}^{\varphi} \nu\right)
$$

The following theorem guarantees that the convolution $\varphi$ is associative.

Theorem 7 For $(\xi, \eta)=\left(\mu, \Phi_{t}^{\varphi} \mu\right) \Subset\left(\nu, \Phi_{t}^{\varphi} \nu\right)$ we have

$$
\eta=\Phi_{t}^{\varphi} \xi
$$

Proof: By the semigroup property $\eta=\varphi_{t_{0}}$ for some $t_{0}$. It remains thus to show that $r_{\eta}(2)=r_{\Phi_{t}^{\varphi}}(2)$. Since $\eta$ is the free convolution of $\Phi_{t}^{\varphi} \mu$ and $\Phi_{t}^{\varphi} \nu$ we have the relation

$$
R_{\eta}(z)=R_{\Phi_{t}^{\varphi} \mu}(z)+R_{\Phi_{t}^{\varphi} \nu}(z)=t\left(r_{\mu}(2)+r_{\nu}(2)\right) R_{\varphi}(z),
$$

hence $\eta=\varphi_{t\left(r_{\mu}(2)+r_{\nu}(2)\right)}$. On the other hand for the measure $\xi$ we have

$$
r_{\xi}(2)=R_{(\xi, \eta)}(2)=R_{\left(\mu, \Phi_{t}^{\varphi} \mu\right)}(2)+R_{\left(\nu, \Phi_{t}^{\varphi} \nu\right)}(2)=r_{\mu}(2)+r_{\nu}(2)
$$

Thus we have $\Phi_{t}^{\varphi} \xi=\eta$.

Now we are going to prove a general form of the central limit theorem for the $\varphi$-convolution. Later on we shall present this theorem in an explicit form for some important examples. As mentioned in the introduction, the central limit theorem is not immediate from the general theorem of [BLS], because the dilation of measures does not commute with that transformation, moreover, the second measure does not have mean zero. First, we are going to prove a technical lemma

Lemma 3 Let $\mu$ be a compactly supported probability measure on the real line with mean zero and variance equal to 1. Let $\varphi$ be a compactly supported $\boxplus$-infinitely divisible measure with mean $\alpha$. Then

$$
\begin{aligned}
R_{\left(\mathcal{D}_{\lambda} \mu, \varphi_{t \lambda^{2}}\right)} & (1)=0, \\
R_{\left(\mathcal{D}_{\lambda} \mu, \varphi_{t \lambda^{2}}\right)}(2) & =\lambda^{2}, \\
R_{\left(\mathcal{D}_{\lambda} \mu, \Phi_{t}^{\varphi} \mathcal{D}_{\lambda} \mu\right)}(n) & =o\left(\lambda^{2}\right) \text { for } n \geq 3 .
\end{aligned}
$$

Proof: By the relation between dilation of a measure and moments (9) the pair

$$
\left(\mathcal{D}_{\lambda} \mu, \Phi_{t}^{\varphi} \mathcal{D}_{\lambda} \mu\right)=\left(\mathcal{D}_{\lambda} \mu, \varphi_{t \lambda^{2}}\right)
$$

has mean $\left(0, t \alpha \lambda^{2}\right)$ and variance of $\mathcal{D}_{\lambda} \mu$ equals $\lambda^{2}$. Moreover we have

$$
r_{\Phi_{t}^{\varphi} \mathcal{D}_{\lambda} \mu}(k)=t r_{\mathcal{D}_{\lambda} \mu}(2) r_{\varphi}(k)=t \lambda^{2} r_{\varphi}(k),
$$

and by moment-cumulant formula for the conditionally free convolution (7) we obtain

$$
\begin{aligned}
& R_{(\mu, \nu)}(1)=r_{\mu}(1)=m_{\mu}(1), \\
& R_{(\mu, \nu)}(2)=r_{\mu}(2)=m_{\mu}(2)-m_{\mu}(1)^{2}, \\
& R_{(\mu, \nu)}(3)=m_{\mu}(3)-2 m_{\mu}(2) m_{\mu}(1)-m_{\mu}(2) m_{\nu}(1)+m_{\mu}(1)^{3}+m_{\mu}(1)^{2} m_{\nu}(1) .
\end{aligned}
$$

hence

$$
\begin{aligned}
& R_{\left(\mathcal{D}_{\lambda} \mu, \varphi_{t \lambda^{2}}\right)}(1)=\lambda m_{\mu}(1)=0, \\
& R_{\left(\mathcal{D}_{\lambda} \mu, \varphi_{t \lambda^{2}}\right)}(2)=\lambda^{2}\left(m_{\mu}(2)-m_{\mu}(1)^{2}\right)=\lambda^{2}, \\
& R_{\left(\mathcal{D}_{\lambda} \mu, \varphi_{t \lambda^{2}}\right)}(3)=\lambda^{3} m_{\mu}(3)-t \lambda^{2} \alpha \lambda^{2}=o\left(\lambda^{2}\right) .
\end{aligned}
$$

Moreover, for $n \geq 3$ we obtain

$$
R_{\left(\mathcal{D}_{\lambda} \mu, \varphi_{t \lambda^{2}}\right)}(n)=o\left(\lambda^{2}\right)
$$


Indeed, if $n \geq 4$ we have two possibilities: either in $\pi \in N C(n)$ there exists an inner block $B_{i}$, or all blocks are outer. If in $\pi \in N C(n)$ there is an inner block $B_{i}$ then also there exists an outer block $B_{j}$ such that $\left|B_{j}\right| \geq 2$. Hence

$$
\begin{aligned}
r_{\varphi_{t \lambda^{2}}}\left(\left|B_{i}\right|\right) & =t \lambda^{2} r_{\varphi}\left(\left|B_{i}\right|\right), \\
R_{\left(\mathcal{D}_{\lambda} \mu, \varphi_{t \lambda^{2}}\right)}\left(\left|B_{j}\right|\right) & =o(\lambda) .
\end{aligned}
$$

By the moment-cumulant formula (7)

$$
\begin{aligned}
& \sum_{\substack{\pi \in \mathrm{NC}(n) \\
\pi \neq(1, \ldots, n)}}\left(\prod_{\substack{B_{i} \in \pi \\
B_{i} \text {-inner }}} R_{\varphi_{t \lambda^{2}}}\left(\left|B_{i}\right|\right)\right)\left(\prod_{\substack{B_{j} \in \pi \\
B_{j} \text { - outer }}} R_{\left(\mathcal{D}_{\lambda} \mu, \varphi_{t \lambda^{2}}\right)}\left(\left|B_{j}\right|\right)\right) \\
= & \sum_{\substack{\pi \in \mathrm{NC}(n) \\
\pi \neq(1, \ldots, n)}}\left(\prod_{\substack{B_{i} \in \pi \\
B_{i} \text {-inner }}} t \lambda^{2} r_{\varphi}\left(\left|B_{i}\right|\right)\right)\left(\prod_{\substack{B_{j} \in \pi \\
B_{j} \text { - outer }}} o(\lambda)\right)=o\left(\lambda^{3}\right)
\end{aligned}
$$

If all the blocks are outer, there must be at least two blocks, $\left|B_{1}\right|+\left|B_{2}\right| \geq 4$, hence

$$
\prod_{B_{j} \in \pi} R_{\left(\mathcal{D}_{\lambda} \mu, \varphi_{t \lambda^{2}}\right)}\left(\left|B_{j}\right|\right)=o\left(\lambda^{3}\right)
$$

because if there exist singletons in $\pi$, the above product equals zero and for the 'worst' case of two blocks consisting of two elements, one has the estimation $\lambda^{2} \cdot \lambda^{2}=o\left(\lambda^{3}\right)$. Therefore

$$
\begin{aligned}
R_{\left(\mathcal{D}_{\lambda} \mu, \varphi_{t \lambda^{2}}\right)}(n) & =m_{\mathcal{D}_{\lambda} \mu}(n)-\sum_{\substack{\pi \in \mathrm{NC}(n) \\
\pi \neq(1, \ldots, n)}}\left(\prod_{\substack{B_{i} \in \pi \\
B_{i} \text {-inner }}} R_{\varphi_{t \lambda^{2}}}\left(\left|B_{i}\right|\right)\right)\left(\prod_{\substack{B_{j} \in \pi \\
B_{j} \text { - outer }}} R_{\left(\mathcal{D}_{\lambda} \mu, \varphi_{t \lambda^{2}}\right)}\left(\left|B_{j}\right|\right)\right) \\
& =\lambda^{n} m_{\mu}(n)-\sum_{\substack{\pi \in \mathrm{NC}(n) \\
\pi \neq(1, \ldots, n)}} o\left(\lambda^{3}\right)=o\left(\lambda^{2}\right) .
\end{aligned}
$$

Theorem 8 (Central limit theorem) Let $\mu$ be a compactly supported probability measure on the real line with mean zero and variance equal to 1 . Let $\varphi$ be a compactly supported $\boxplus$-infinitely divisible measure with mean $\alpha$. Then the sequence

$$
\mathcal{D}_{1 / \sqrt{N}} \mu \varphi \ldots \varphi \mathcal{D}_{1 / \sqrt{N}} \mu=\left(\mathcal{D}_{1 / \sqrt{N}} \mu, \Phi_{t}^{\varphi} \mathcal{D}_{1 / \sqrt{N}} \mu\right) 匹 \ldots \text { c. }\left(\mathcal{D}_{1 / \sqrt{N}} \mu, \Phi_{t}^{\varphi} \mathcal{D}_{1 / \sqrt{N}} \mu\right)
$$

is $*$-weakly convergent as $N \rightarrow \infty$ to the measure $\xi$, such that $\varphi_{t}=\Phi_{t}^{\varphi} \xi$ and

$$
R_{\left(\xi, \varphi_{t}\right)}(z)=z \text {. }
$$

Proof: Since both measures have compact support it suffices to check that the pair of cumulants

$$
\left(R_{\left(\mathcal{D}_{1 / \sqrt{N}} \mu, \Phi_{t}^{\varphi} \mathcal{D}_{1 / \sqrt{N}} \mu\right)}(n), r_{\Phi_{t}^{\varphi} \mathcal{D}_{1 / \sqrt{N}} \mu}(n)\right)
$$

converge to the cumulants of the limiting measures, defined by

$$
R_{\left(\xi, \varphi_{t}\right)}(n)= \begin{cases}0, & n \neq 2 \\ 1, & n=2\end{cases}
$$

From Lemma 3 we obtain

$$
\begin{aligned}
& R_{\left(\mathcal{D}_{1 / \sqrt{N}} \mu, \Phi_{t}^{\varphi} \mathcal{D}_{1 / \sqrt{N}} \mu\right)}(1)=0 \\
& R_{\left(\mathcal{D}_{1 / \sqrt{N}} \mu, \Phi_{t}^{\varphi} \mathcal{D}_{1 / \sqrt{N}} \mu\right)}(2)=\frac{1}{N} \\
& R_{\left(\mathcal{D}_{1 / \sqrt{N}} \mu, \Phi_{t}^{\varphi} \mathcal{D}_{1 / \sqrt{N}} \mu\right)}(k)=o\left(\frac{1}{N}\right), \quad k \geq 3 .
\end{aligned}
$$


Now we take $\lambda=\frac{1}{\sqrt{N}}$ and denote

$$
\left(\mu_{N}, \Phi_{t}^{\varphi} \mu_{N}\right)=\left(\mathcal{D}_{1 / \sqrt{N}} \mu, \Phi_{t}^{\varphi} \mathcal{D}_{1 / \sqrt{N}} \mu\right) 匹 \ldots \square\left(\mathcal{D}_{1 / \sqrt{N}} \mu, \Phi_{t}^{\varphi} \mathcal{D}_{1 / \sqrt{N}} \mu\right)
$$

Hence

$$
\begin{aligned}
& R_{\left(\mu_{N}, \Phi_{t}^{\varphi} \mu_{N}\right)}(1)=0, \\
& R_{\left(\mu_{N}, \Phi_{t}^{\varphi} \mu_{N}\right)}(2)=N \cdot \frac{1}{N}=1, \\
& R_{\left(\mu_{N}, \Phi_{t}^{\varphi} \mu_{N}\right)}(k)=N \cdot o\left(\frac{1}{N}\right) \rightarrow_{N \rightarrow \infty} 0, \quad k \geq 3,
\end{aligned}
$$

and therefore

$$
R_{\left(\mu_{N}, \Phi_{t}^{\varphi} \mu_{N}\right)}(z) \rightarrow R_{\left(\xi, \varphi_{t}\right)}(z)=z
$$

because of

$$
\left(\Phi_{t}^{\varphi} \mathcal{D}_{1 / \sqrt{N}} \mu\right)^{\boxplus N}=\left(\varphi_{t / N}\right)^{\boxplus N}=\varphi_{t}
$$

By the relations (24), (25), (26), we are able to calculate first and second moments of the measure $\xi$. Indeed,

$$
\begin{aligned}
& m_{\xi}(1)=R_{\left(\xi, \varphi_{t}\right)}(1)=0, \\
& m_{\xi}(2)=R_{\left(\xi, \varphi_{t}\right)}(2)=1, \\
& m_{\xi}(3)=R_{\left(\xi, \varphi_{t}\right)}(3)+m_{\xi}(2) m_{\varphi}(1)=\alpha .
\end{aligned}
$$

We shall now prove the Poisson limit theorem for the $\varphi$ convolution.

Theorem 9 For $\lambda>0$ define for all $N$

$$
\mu_{N}=\left(1-\frac{\lambda}{N}\right) \delta_{0}+\frac{\lambda}{N} \delta_{1}, \quad N \geq 1
$$

Then we have

$$
w^{*}-\lim _{N \rightarrow \infty}\left(\mu_{N}, \Phi_{t}^{\varphi} \mu_{N}\right) \llbracket \ldots \llbracket\left(\mu_{N}, \Phi_{t}^{\varphi} \mu_{N}\right)=\left(p_{\lambda}, \varphi_{\lambda t}\right),
$$

where

$$
R_{\left(p_{\lambda}, \varphi_{\lambda t}\right)}(z)=\frac{\lambda}{1-z}
$$

Proof: By definition we have

$$
\Phi_{t}^{\varphi} \mu_{N}=\varphi_{t r_{\mu_{N}}(2)}=\varphi_{t\left(\frac{\lambda}{N}-\left(\frac{\lambda}{N}\right)^{2}\right)},
$$

hence

$$
r_{\Phi_{t}^{\varphi} \mu_{N}}(n)=t\left(\frac{\lambda}{N}-\left(\frac{\lambda}{N}\right)^{2}\right) r_{\varphi}(n)=\frac{\lambda}{N} t r_{\varphi}(n)+o\left(\frac{\lambda}{N}\right)
$$

and therefore

$$
N \cdot r_{\Phi_{t}^{\varphi} \mu_{N}}(n)=N\left(\frac{\lambda}{N} t r_{\varphi}(n)+o\left(\frac{\lambda}{N}\right)\right)=\lambda t r_{\varphi}(n)=r_{\varphi_{\lambda t}}(n)
$$

Since

$$
m_{\mu_{N}}(n)=\frac{\lambda}{N}
$$


we have

$$
R_{\left(\mu_{N}, \Phi_{t}^{\varphi} \mu_{N}\right)}(n)=\frac{\lambda}{N}+o\left(\frac{\lambda}{N}\right)
$$

Hence it follows, that in the limit we have measures $\left(p_{\lambda}, \varphi_{\lambda t}\right)$ and the measure $p_{\lambda}$ is determined by

$$
R_{\left(p_{\lambda}, \varphi_{\lambda t}\right)}(n)=\lambda,
$$

hence

$$
R_{\left(p_{\lambda}, \varphi_{\lambda t}\right)}(z)=\frac{\lambda}{1-z}
$$

In the particular case, when $\varphi$ equals standard Wigner law $\omega$ or $\varphi=\pi_{1}$-the free Poisson law with intensity 1 , we are able to calculate the central limiting and Poisson measures explicitly.

Definition 5 In the particular case when $\varphi$ equals the standard Wigner law $\omega$, i.e. $d \omega(x)=\frac{1}{2 \pi} \sqrt{4-x^{2}} d x$, we define a new Wigner convolution by $\xi=\mu \omega \nu$ where $(\xi, \eta)=\left(\mu, \Phi_{t}^{\omega} \mu\right) \square\left(\nu, \Phi_{t}^{\omega} \nu\right)$.

Definition 6 In the case when $\varphi$ equals the standard free Poisson law $\pi$, we define a new Poisson convolution by $\xi=\mu \pi \nu$ where $(\xi, \eta)=\left(\mu, \Phi_{t}^{\pi} \mu\right) \square\left(\nu, \Phi_{t}^{\pi} \nu\right)$.

Remark 8 The Wigner convolution $\omega$ for the specific choice $t=1$ has been introduced by Oravecz as an example of a pure convolution, see [O1, O2].

Theorem 10 (Central limit theorem for the $\omega$ convolution) Let $\mu$ be a probability measure on the real line with the mean zero and variance equal to 1 . Then the sequence

$$
\mathcal{D}_{1 / \sqrt{N}} \mu \omega \ldots \omega \mathcal{D}_{1 / \sqrt{N}} \mu=\left(\mathcal{D}_{1 / \sqrt{N}} \mu, \Phi_{t}^{\omega} \mathcal{D}_{1 / \sqrt{N}} \mu\right) 匹 \ldots\left(\mathcal{D}_{1 / \sqrt{N}} \mu, \Phi_{t}^{\omega} \mathcal{D}_{1 / \sqrt{N}} \mu\right)
$$

is $*$-weakly convergent to the Kesten measure $\kappa_{t}$, which has a part absolutely continuous with respect to the Lebesgue measure, denoted $\tilde{\kappa}_{t}$ and for $t<\frac{1}{2}$ a discrete part $\hat{\kappa}_{t}$ with two atoms:

$$
\begin{aligned}
d \tilde{\kappa}_{t}(x) & =\frac{1}{2 \pi} \cdot \frac{\sqrt{4 t-x^{2}}}{1-(1-t) x^{2}} \chi_{[-2 \sqrt{t}, 2 \sqrt{t}]}(x) d x \\
\hat{\kappa}_{t} & =\frac{1-2 t}{2-2 t}\left(\delta_{-\frac{1}{\sqrt{1-t}}}+\delta_{\frac{1}{\sqrt{1-t}}}\right) \quad \text { for } t<\frac{1}{2} .
\end{aligned}
$$

Proof: By Theorem 8 the limiting measure $\xi$ is determined by

$$
R_{\left(\xi, \omega_{t}\right)}(z)=z
$$

and by

$$
\frac{1}{G_{\omega_{t}}(z)}=z-t G_{\omega_{t}}(z)
$$

one can obtain

$$
G_{\omega_{t}}(z)=\frac{z-\sqrt{z^{2}-4 t}}{2 t}
$$

hence

$$
\begin{aligned}
& \frac{1}{G_{\xi}(z)}=z-\frac{z-\sqrt{z^{2}-4 t}}{2 t} \\
& G_{\xi}(z)=\frac{2 t}{2 t z-z+\sqrt{z^{2}-4 t}}=\frac{(1-2 t) z+\sqrt{z^{2}-4 t}}{2(1-t) z-2} .
\end{aligned}
$$

Hence $\xi$ equals the Kesten measure $\kappa_{t}$. By [BW2, W] one can find the measure and the continued fraction representation of $G_{\xi}(z)$. 
Theorem 11 (Poisson limit theorem for the $\omega$ convolution) The Poisson measure $p_{\lambda}$ for $\omega$ convolution is of the form $p_{\lambda}=\tilde{p_{\lambda}}+\hat{p_{\lambda}}$, where $p_{\lambda}^{c}$ is the continuous part with density

$$
d \tilde{p_{\lambda}}(t)=\frac{1}{2 \pi} \frac{-\lambda \sqrt{4 t \lambda-x^{2}}}{\left(x^{3}-x^{2}(1+\lambda+t \lambda)+2 t x \lambda^{2}-t \lambda^{3}\right)} \chi_{[-2 \sqrt{\lambda t}, 2 \sqrt{\lambda t}]} d t
$$

and $\hat{p_{\lambda}}$ is an atom in the real zero $x_{0}$ of the polynomial

$$
z^{3}-z^{2}(1+\lambda+t \lambda)+2 t z \lambda^{2}-t \lambda^{3}
$$

with weight equal to the residue of the Cauchy transform $G_{p_{\lambda}}(z)$ in $x_{0}$. The actual dependence of this weight on $\lambda$ and $t$ is rather complicated.

Proof: By Theorem 9 the limiting measure $p_{\lambda}$ is determined by the relation

$$
R_{\left(p_{\lambda}, \omega_{\lambda t}\right)}(z)=\frac{\lambda}{1-z}
$$

and because of

$$
G_{\omega_{\lambda t}}(z)=\frac{z-\sqrt{z^{2}-4 \lambda t}}{2 \lambda t}
$$

we obtain

$$
\frac{1}{G_{p_{\lambda}}(z)}=z-\frac{2 \lambda^{2} t}{2 \lambda t-z+\sqrt{z^{2}-4 \lambda t}}=\frac{z^{2}-2 z t \lambda+2 t \lambda^{2}-z \sqrt{z^{2}-4 t \lambda}}{z-2 t \lambda-\sqrt{z^{2}-4 t \lambda}} .
$$

Hence

$$
G_{p_{\lambda}}(z)=\frac{z-2 t \lambda-\sqrt{z^{2}-4 t \lambda}}{z^{2}-2 z t \lambda+2 t \lambda^{2}-z \sqrt{z^{2}-4 t \lambda}}=\frac{2 z^{2}-z(2+\lambda+2 t \lambda)+\lambda\left(2 t \lambda+\sqrt{z^{2}-4 t \lambda}\right)}{2\left(z^{3}-z^{2}(1+\lambda+t \lambda)+2 t \lambda^{2} z-t \lambda^{3}\right)} .
$$

Because a denominator of the Cauchy transform of the measure $p_{\lambda}$ has only one real root $x_{0}$, the measure $p_{\lambda}$ has only one atom $\delta_{x_{0}}$ with respective weight, which is equal to the residue $G_{p_{\lambda}}$ in the point $x_{0}$. Moreover, by Proposition 1 one can find that $x_{0}$ is the unique singular part of the measure $p_{\lambda}$. The density $f_{p_{\lambda}}(x), x \in \mathbb{R}$ of the absolute continuous part of the measure $p_{\lambda}$ can now be calculated by the Stieltjes formula

$$
f_{p_{\lambda}}(x)=-\frac{1}{\pi} \lim _{\epsilon \rightarrow 0^{+}} \operatorname{Im} G_{p_{\lambda}}(x+i \epsilon)=\frac{1}{2 \pi} \frac{-\lambda \sqrt{4 t \lambda-x^{2}}}{\left(x^{3}-x^{2}(1+\lambda+t \lambda)+2 t x \lambda^{2}-t \lambda^{3}\right)}
$$

for $x \in[-2 \sqrt{\lambda t}, 2 \sqrt{\lambda t}]$. By the relation (7) we can calculate, that

$$
\begin{aligned}
& m_{p_{\lambda}}(1)=\lambda \\
& m_{p_{\lambda}}(2)=\lambda+\lambda^{2}, \\
& m_{p_{\lambda}}(3)=\lambda+2 \lambda^{2}+\lambda^{3} \\
& m_{p_{\lambda}}(4)=\lambda+(3+t) \lambda^{2}+3 \lambda^{3}+\lambda^{4}, \\
& m_{p_{\lambda}}(5)=\lambda+(4+2 t) \lambda^{2}+(6+2 t) \lambda^{3}+4 \lambda^{4}+\lambda^{5}, \\
& m_{p_{\lambda}}(6)=\lambda+(5+3 t) \lambda^{2}+\left(10+6 t+2 t^{2}\right) \lambda^{3}+(10+3 t) \lambda^{4}+5 \lambda^{5}+\lambda^{6}, \\
& m_{p_{\lambda}}(7)=\lambda+(6+4 t) \lambda^{2}+\left(15+12 t+5 t^{2}\right) \lambda^{3}+\left(20+12 t+4 t^{2}\right) \lambda^{4}+(15+4 t) \lambda^{5}+6 \lambda^{6}+\lambda^{7} .
\end{aligned}
$$

Moreover, we obtain the following continued fraction form of the Cauchy transform of the measure $p_{\lambda}$ :

$$
G_{p_{\lambda}}(z)=\frac{1}{z-\lambda-\frac{\lambda}{z-1-\frac{t \lambda}{z-\frac{t \lambda}{z-\frac{t \lambda}{z-\ddots} .}}} .}
$$


Remark 9 Polynomials orthogonal with respect to measures of the form $\frac{\sqrt{1-x^{2}}}{\rho(x)}$, where $\rho(x)$ is a polynomial, were first considered by Bernstein, see [Sz].

Theorem 12 (Central limit theorem for the $\pi$-convolution) Let $\mu$ be a probability measure on the real line with the mean zero and variance equal to 1 . Then the sequence

$$
\mathcal{D}_{1 / \sqrt{N}} \mu \pi \ldots \pi \mathcal{D}_{1 / \sqrt{N}} \mu=\left(\mathcal{D}_{1 / \sqrt{N}} \mu, \Phi_{t}^{\pi} \mathcal{D}_{1 / \sqrt{N}} \mu\right) 匹 \ldots \text { 띠 }\left(\mathcal{D}_{1 / \sqrt{N}} \mu, \Phi_{t}^{\pi} \mathcal{D}_{1 / \sqrt{N}} \mu\right)
$$

is $*$-weakly convergent to the measure $\xi$, such that

$$
\xi(x)=\frac{1}{2 \pi} \frac{\sqrt{4 t-(1-x+t)^{2}}}{x^{3}-x^{2}+(t-1) x+1} \chi_{\left[(1-\sqrt{t})^{2},(1+\sqrt{t})^{2}\right]} d x+\operatorname{Res}\left(x_{0}\right) \delta_{x_{0}},
$$

where

$$
\begin{aligned}
Q & =-16-9 t+3 \sqrt{96 t-39 t^{2}+12 t^{3}} \\
x_{0} & =\frac{1}{3}\left(1+\frac{\sqrt[3]{2}(4-3 t)}{\left.\sqrt[3]{Q}+\frac{\sqrt[3]{Q}}{\sqrt[3]{2}}\right)}\right. \\
\operatorname{Res}\left(x_{0}\right) & =\frac{\sqrt[3]{4}\left(16-35 t+12 t^{2}\right)+2 \sqrt[3]{Q^{2}}(2-t)-8 \sqrt[3]{2 Q}(1+t)+(\sqrt[3]{4}+2 \sqrt[3]{2 Q}) \sqrt{96 t-39 t^{2}+12 t^{3}}}{Q \sqrt[3]{2 Q}+\sqrt[3]{Q^{2}}(8-6 t)+2 \sqrt[3]{4}(4-3 t)^{2}} .
\end{aligned}
$$

Proof: Using Theorem 8 we obtain that the limiting measure $\xi$ satisfies the relation

$$
R_{\left(\xi, \pi_{t}\right)}(z)=z \text {. }
$$

Because of

$$
\frac{1}{G_{\pi_{t}}(z)}=z-\frac{t}{1-G_{\pi_{t}}(z)}
$$

which gives

$$
G_{\pi_{t}}(z)=\frac{1+z-t-\sqrt{(1+z-t)^{2}-4 z}}{2 z}=\frac{1+z-t-\sqrt{(1-z+t)^{2}-4 t}}{2 z}
$$

we obtain

$$
\begin{aligned}
& \frac{1}{G_{\xi}(z)}=z-\frac{1+z-t-\sqrt{(1-z+t)^{2}-4 t}}{2 z}=\frac{2 z^{2}-1-z+t+\sqrt{(1-z+t)^{2}-4 t}}{2 z} \\
& G_{\xi}(z)=\frac{2 z}{2 z^{2}-1-z+t+\sqrt{(1-z+t)^{2}-4 t}}=\frac{2 z^{2}-z-1+t-\sqrt{(1-z+t)^{2}-4 t}}{2\left(z^{3}-z^{2}+(t-1) z+1\right)} .
\end{aligned}
$$

The appropriate choice of the branch of a square root gives in limit for real $z=x$

$$
\sqrt{(1-z+t)^{2}-4 t}= \begin{cases}\sqrt{(1-x+t)^{2}-4 t} & \text { for } x \geq(1+\sqrt{t})^{2} \\ -\sqrt{(1-x+t)^{2}-4 t} & \text { for } x \leq(1-\sqrt{t})^{2} \\ i \sqrt{4 t-(1-x+t)^{2}} & \text { for }(1-\sqrt{t})^{2}<x<(1+\sqrt{t})^{2}\end{cases}
$$

Because one can decompose a denominator of the Cauchy transform

$$
\left(z^{3}-z^{2}+(t-1) z+1\right)=\left(z-x_{0}\right)\left(z^{2}+b z+c\right),
$$

where

$$
\begin{aligned}
Q & =-16-9 t+3 \sqrt{96 t-39 t^{2}+12 t^{3}} \\
x_{0} & =\frac{1}{3}\left(1+\frac{\sqrt[3]{2}(4-3 t)}{\left.\sqrt[3]{Q}+\frac{\sqrt[3]{Q}}{\sqrt[3]{2}}\right)}\right. \\
b & =\frac{2 \sqrt[3]{2}(4-3 t)-4 \sqrt[3]{Q}+\sqrt[3]{4 Q^{2}}}{6 \sqrt[3]{Q}}, \\
c & =\frac{-\sqrt[3]{4} Q+Q \sqrt[3]{2 Q}+2 \sqrt[3]{4}(4-3 t)^{2}-6 \sqrt[3]{Q^{2}}(1-t)-2 \sqrt[3]{2 Q}(4-3 t)}{18 \sqrt[3]{Q^{2}}}
\end{aligned}
$$


and $x_{0}$ is the single real zero of the denominator, to work out the residue in $x_{0}$ it suffices to find $2 x_{0}^{2}-x_{0}-1+t-$ $\sqrt{\left(1-x_{0}+t\right)^{2}-4 t}$ and $\left(x_{0}^{2}+b x_{0}+c\right)$.

Moreover, since $x_{0} \leq 0 \leq(1-\sqrt{t})^{2}$, then because $x_{0}$ is also a zero of $2 z^{2}-1-z+t+\sqrt{(1-z+t)^{2}-4 t}$, we have for $x_{0}$

$$
-\sqrt{\left(1-x_{0}+t\right)^{2}-4 t}=2 x_{0}^{2}-x_{0}-1+t,
$$

and it follows, that the Cauchy transform $G_{\xi}(z)$ has a simple pole in a point $x_{0}$ with residue

$$
\operatorname{Res}\left(x_{0}\right)=\frac{\sqrt[3]{4}\left(16-35 t+12 t^{2}\right)+2 \sqrt[3]{Q^{2}}(2-t)-8 \sqrt[3]{2 Q}(1+t)+(\sqrt[3]{4}+2 \sqrt[3]{2 Q}) \sqrt{96 t-39 t^{2}+12 t^{3}}}{Q \sqrt[3]{2 Q}+\sqrt[3]{Q^{2}}(8-6 t)+2 \sqrt[3]{4}(4-3 t)^{2}}
$$

By Proposition 1 one can find that $x_{0}$ is the unique singular part of the measure $\xi$.

The density $f_{\xi}(x), x \in \mathbb{R}$ of the absolute continuous part of the measure $\xi$ can now be calculated by the Stieltjes formula

$$
f_{\xi}(x)=-\frac{1}{\pi} \lim _{\epsilon \rightarrow 0^{+}} \operatorname{Im} G_{\xi}(x+i \epsilon)=\frac{1}{2 \pi} \frac{\sqrt{4 t-(1-x+t)^{2}}}{x^{3}-x^{2}+(t-1) x+1}, \quad \text { where } x \in\left[(1-\sqrt{t})^{2},(1+\sqrt{t})^{2}\right] .
$$

By the relation (7) we can calculate, that

$$
\begin{aligned}
& m_{\xi}(3)=t \\
& m_{\xi}(4)=1+t+t^{2} \\
& m_{\xi}(5)=3 t+3 t^{2}+t^{3} \\
& m_{\xi}(6)=1+3 t+9 t^{2}+6 t^{3}+t^{4} \\
& m_{\xi}(7)=6 t+18 t^{2}+24 t^{3}+10 t^{4}+t^{5}
\end{aligned}
$$

and the measure $\xi$ is not symmetric. Moreover,

$$
G_{\xi}(z)=\frac{1}{z-\frac{1}{z-t-\frac{t}{z-1-t-\frac{t}{z-1-t-\frac{t}{z-1-t-\ddots}}}} .}
$$

A diagram of this measure for $t=1$ is presented on the following figure, the vertical line indicates the atom.

Figure 1: Density of the central limit measure for the Poisson convolution

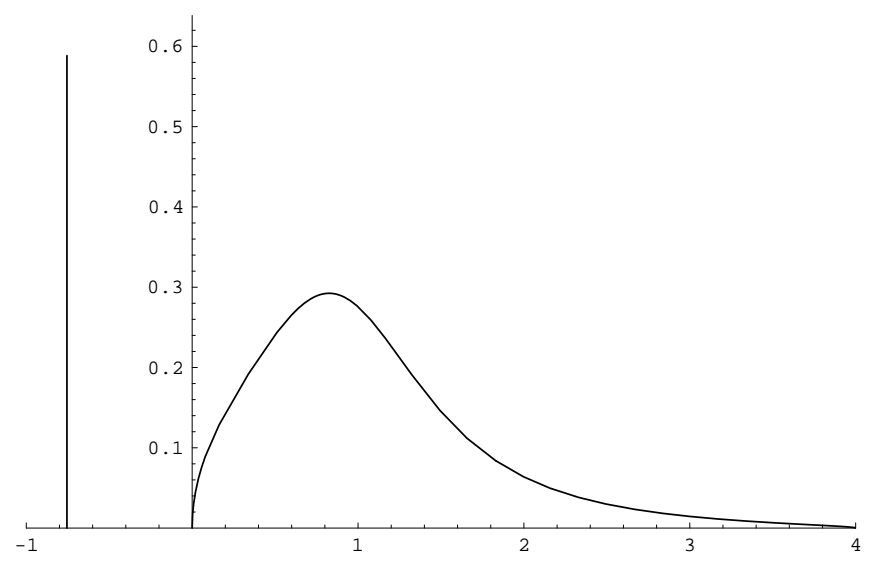


Theorem 13 (Poisson limit theorem for the $\pi$ convolution) The Poisson measure $p_{\lambda}$ for the convolution $\pi$ is equal to the conditionally free Poisson law, i.e. is of the form

$$
p_{\lambda}=a \delta_{0}+b \delta_{z_{0}}+\tilde{\pi}_{\lambda, \lambda t}
$$

with

$$
\begin{aligned}
a & =\frac{1-\lambda t}{1+\lambda-\lambda t} \max (1-\lambda t, 0), \\
b & =\frac{t z_{0}-\lambda}{z_{0}(1-t)} \max \left(\lambda(1-t)^{2}-t, 0\right), \\
z_{0} & =\lambda+\frac{1}{1-t}, \\
\tilde{\pi}_{\lambda, \lambda t}(x) & =\frac{1}{2 \pi x} \frac{\sqrt{4 \lambda t-(x-(1+\lambda t))^{2}}}{x(t-1)+(1-\lambda t+\lambda)} .
\end{aligned}
$$

Proof: Indeed, by the Theorem 9 we obtain

$$
\frac{1}{G_{p_{\lambda}}(z)}=z-R_{\left(p_{\lambda}, \pi_{\lambda t}\right)}\left(G_{\pi_{\lambda t}}(z)\right)
$$

and because of

$$
G_{\pi_{\lambda t}}(z)=\frac{z+1-\lambda t-\sqrt{(z+1-\lambda t)^{2}-4 z}}{2 z}
$$

we have

$$
\begin{aligned}
\frac{1}{G_{p_{\lambda}}(z)} & =z-\frac{2 \lambda z}{z-1+\lambda t+\sqrt{(z-1-\lambda t)^{2}-4 \lambda t}}=\frac{z(z-1-2 \lambda+\lambda t)+z \sqrt{(z-1-\lambda t)^{2}-4 \lambda t}}{z-1+\lambda t+\sqrt{(z-1-\lambda t)^{2}-4 \lambda t}} \\
& =\frac{4 \lambda t z^{2}(z(t-1)+1+\lambda-\lambda t)}{2 \lambda z\left(1-\lambda t+(2 t-1) z+\sqrt{(z-1-\lambda t)^{2}-4 \lambda t}\right)} .
\end{aligned}
$$

Hence

$$
G_{p_{\lambda}}(z)=\frac{1-\lambda t+(2 t-1) z+\sqrt{(z-1-\lambda t)^{2}-4 \lambda t}}{2 z(z(t-1)+1+\lambda-\lambda t)}
$$

and $p_{\lambda}$ is the conditionally free Poisson measure $\pi_{\lambda, \lambda t}$. The continued fraction form of $G_{p_{\lambda}}(z)$ can be found in [BLS].

Remark 10 The coefficients of the continued fraction form of the Cauchy transform for the central and Poisson limit measures of the convolutions $\pi$ and $\omega$ are equal to the respective coefficients of the underlying measure $\varphi=\pi, \omega$ starting from the third level. Our hypothesis is that this property is a universal property of the convolution $\varphi$.

\section{References}

[AB] L.Accardi, M.Bożejko, Interacting Fock spaces and gaussianization of probability measures, Infinite Dimensional Analysis, Quantum Probability and Related Topics, Vol. 1, No. 4 (1998), 663-670

[Ak] N.I.Akhiezer, The classical moment problem, Oliver and Boyd, Edinburgh and London, 1965

[BV] H.Bercovici, D.Voiculescu, Levy-Hincin type theorems for multiplicative and additive free convolution, Pacific J. Math., 153 (1992), 217-248

[BLS] M.Bożejko, M.Leinert, R.Speicher, Convolution and limit theorems for conditionally free random variables, $P a-$ cific Journal of Mathematics, Vol. 175, No. 2 (1996), 357-388

[BW1] M.Bożejko, J.Wysoczański, New examples of convolutions and non-commutative central limit theorems, Banach Center Publications, Vol. 43(1998), 95-103 
[BW2] M.Bożejko, J.Wysoczański, Remarks on $t$-transformations of measures and convolutions, Ann. I. H. Poincaré-PR 37, 6(2001) 737-761

[BrW] W.Bryc, J.Wesołowski: Bi-Poisson Process, Preprint, arXiv:math.PR/0404241, 2004

[KY] A. Krystek, H. Yoshida: Generalized t-transformatons of probability measures and deformed convolution, Probability and Mathematical Statistics vol.24, fasc.1 (2004), 97-119

[Leh] F.Lehner, Cumulants in noncommutative probability theory I, Math.Z., 248 (2004), 67-100

[O1] F.Oravecz, The number of pure convolutions arising from conditionally free convolution, preprint

[O2] F.Oravecz, Pure convolutions arising from conditionally free convolution, preprint

[RS4] M.Reed, B.Simon, Methods of Modern Mathematical Physics, IV, Analysis of Operators, Academic Press, New York, 1978

[SY] N.Saitoh, H.Yoshida, The infinite divisibility and orthogonal polynomials with a constant recursion formula in free probability theory, Probability and Mathematical Statistics Vol. 21, Fasc. 1 (2001), 159-170

[S] R.Speicher, Multiplicative functions on the lattice of non-crossing partitions and free convolution, Math. Ann. 298 (1994), 611-628

[Sz] G.Szegö, Orthogonal Polynomials, AMS Colloquium Publications, vol. 23, Providence, Rhode Island, 4 th ed., 1975

[W] Ł.Wojakowski, Probability Interpolating between Free and Boolean, Ph.D. Thesis, University of Wrocław, 2004 